\title{
High frequency focused imaging for ultrasonic probe integrity inspection
}

\author{
Linas Svilainis $^{\mathrm{a}, *}$, Darius Kybartas ${ }^{\mathrm{a}}$, Arturas Aleksandrovas ${ }^{\mathrm{a}}$, Tomas E.Gomez Alvarez-Arenas ${ }^{\mathrm{b}}$

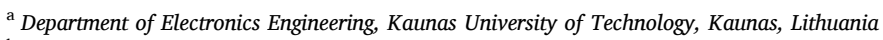 \\ ${ }^{\mathrm{b}}$ Ultrasonic and Sensors Technologies Department, Institute of Physical and Information Technologies (ITEFI), Spanish National Research Council (CSIC), Madrid, Spain
}

\section{A R T I C L E I N F O}

\section{Keywords:}

Ultrasound transducers

Ultrasonic non-destructive testing

Ultrasound imaging

Probe characterization

Reflection imaging

Transmission imaging

\begin{abstract}
A B S T R A C T
Most ultrasonic probes present a layered structure that may comprise delay lines, acoustic lens, wear plates, matching layers, electrodes, piezoelectric layers, and a backing block. Probe integrity can be compromised by different defects, such as deficient bonding, presence of air bubbles or porosity, lack of electrical conductivity in electrodes, and deviations in the layers' acoustic thicknesses, impedances or attenuation values. Conventional testing procedures are based on the integral properties of the probe, such as acoustic directivity, sensitivity, and electrical impedance. An alternative technique for the non-destructive inspection of the probe's integrity is presented in this study. The proposed technique is based on imaging of the internal structure using a higher frequency focused ultrasound. Scanning the probe's surface using this focused beam provides two imaging modes: reflection and transmission. In the reflection mode, the higher intensity of the echoes indicates the presence of the defects, such as disbonds, poor acoustic matching or porosity. The main novelty of the proposed technique is in the transmission mode: the probe's electric response to the high frequency focused beam is used to get a 2D distribution of transduction efficiency. Moreover, the probe's response can be gated to separate, both front and back side, responses in the time domain, so the local integrity of an electroacoustic structure is evaluated. To verify the proposed technique, a focused $20 \mathrm{MHz}$ transducer with a focal spot diameter of $0.35 \mathrm{~mm}$ was mounted on an ultrasonic scanner and immersed in water above the surface of the probe to be tested. Signals were gated by the layers' thickness, giving the C-scan images corresponding to the different material interfaces inside the tested probes: front face, matching layers, piezoelement and backing. It was demonstrated that the resulting C-scan images are informative in evaluating probe's integrity and layers' acoustic thickness. Comparison of the C-scan images obtained in the reflection and transmission modes shows a good agreement in the structure deficiencies evaluation. The technique proposed is attractive due to its simplicity. It can be useful in several applications: i) the probes already in use can be verified and if any deficiencies are detected, the information obtained can be used to evaluate and guide repair; ii) fabrication quality can be verified by confirming the adhesion of layers and parameters in 2D; and iii) the development of new fabrication routes can be guided, where adherence, compatibility, homogeneity, etc. of new materials or new technologies can be evaluated.
\end{abstract}

\section{Introduction}

The continuously increasing use of ultrasound in many different fields has a significant impact on the variety and volume of the commercially available probes. The quality of the probes is usually evaluated by the parameters that are defined in the standards [1]. The manufacturer has to specify the pulse shape in time and frequency domains, center frequency, relative bandwidth, pulse-echo sensitivity, distance-amplitude curve, impedance, and static capacitance of the probe [2]. The angle of divergence, beam axis offset, squint angle, focal width, and focal length are also usually specified. It is expected that the transducer parameters are as specified when the probe is reaching the final user. The standards also define how frequently probes have to be checked to confirm that there is no degradation in their performance. Probe index, beam angle, physical state and external aspects have to be checked daily. Sensitivity and pulse duration are checked weekly or every time the equipment is used again [3]. Sensitivity testing is simple: the reflection from a target in water (immersion transducers) or from a reference block (contact) is measured. Variation in sensitivity must be within $6 \mathrm{~dB}$. While such a procedure is simple and fast, it does not ensure the transducer integrity. Many ultrasonic transducers have a multilayered structure made of protective, matching, electrodes, piezoelectric,

\footnotetext{
* Corresponding author.

E-mail address: linas.svilainis@ktu.lt (L. Svilainis).
} 


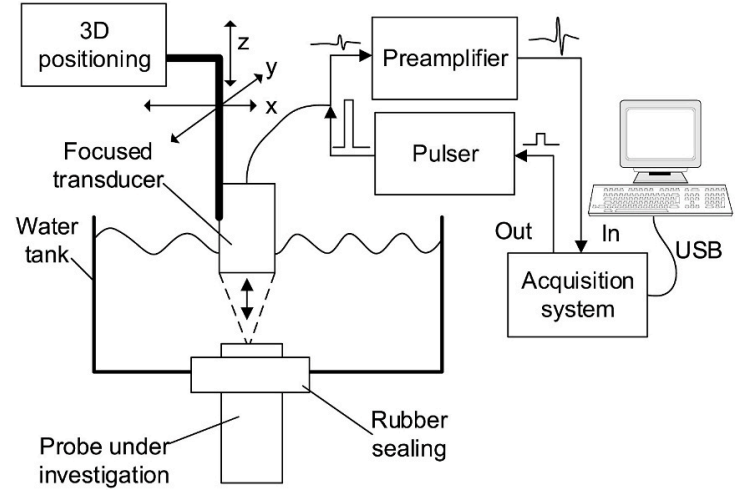

Fig. 1. Reflection imaging setup.

and damping layers. Some of them have frequency-matched thicknesses and are made of materials with specific values of both acoustic impedance and attenuation coefficient [4,5]. Bonding between the layers, homogeneity of the layers and their acoustic thickness accuracy are the factors affecting the final performance. Local disbonds or thickness variations could be hard to notice if a transducer performance is evaluated by the static capacitance, pulse shape or frequency response measurements [6,7]. Furthermore, while in design or manufacturing technology adjustment stage, or even in production, it is desirable to spot even the slightest discrepancies in performance [8].

Monitoring the transducer electrical impedance changes $[9,10]$ or acoustic directivity tests $[1,11,12]$ are the usual techniques. Yet, these approaches are based on global properties and are not sensitive to local defects or disbonds in the layers of the transducer. Moreover, they do not provide any information about the origin of the problem. Hydrophone based scanning is helpful for the evaluation of the distribution of the acoustic pressure in the near field [13]. Surface vibrations can be monitored using laser vibrometry [14] to locate possible defects, but this requires specific laser instrumentation which is complex to operate and expensive. A completely different technique is based on the evaluation of the transducers by their B-scan images [15] or air reverberations [16], but this is valid only for the rough estimation of defects. Nevertheless, these techniques do not reveal the presence of local defects so such an inspection might miss the faulty probe. Transducer development and production tuning involve application of several layers, contacts and backing installation. It is desirable to have the confirmation that the whole layered structure is intact.

Non-destructive testing (NDT) of layered structures received a lot of attention both in recent research $[17,18]$ and background development [19-24]. This technique can be applied for the transducer layers integrity inspection. A focused high frequency transducer can be used to inspect the structure of the probe in a reflection mode. The reflections received would originate at the probe's front face, the inner interfaces between matching layers, piezoelement, and backing. When properly gated, the signals received can be used for the transducer integrity inspection. This approach was used as a reference in our investigation. However, it requires careful alignment of the scanning and inspection surfaces; special signal processing needs to be applied for the final alignment of signals; signals can overlap, so spectral or temporal decomposition techniques have to be used.

In this paper, we propose a different approach based on the same experimental setup as above, where the main difference is that now the electrical signal generated by the tested probe is used to evaluate the sensitivity at each surface point hit by a focused beam, producing a 2D sensitivity map after the point-by-point scanning.

Section 2 presents the conventional approach, where the reflection mode imaging is used. Section 3 presents the proposed approach, using a transmission mode. Section 4 presents experimental data where both reflection and transmission modes are used for the integrity assessment

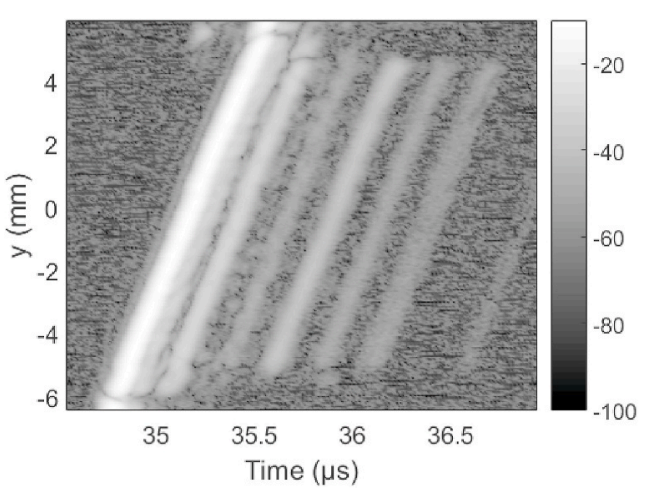

Fig. 2. An example of the B-scan of the received reflections (grey scale is dBV).

of various probes.

\section{Conventional approach: reflection imaging}

The probe under the investigation is assumed to be relatively low frequency (1-5 MHz), compared with the testing probe ( $20 \mathrm{MHz})$. These frequencies are commonly used in NDT as a near optimum balance is achieved between penetration capability, structural noise and resolution. The tested probe is mounted into the bottom of the water tank, exposing the front face for the pulse-echo (reflection) probing of the inner layers (Fig. 1). This transducer is addressed as "the probe" for the rest of the text. The focused transducer used to test this probe is selected so that it has a significantly higher center frequency than the probe, is positioned above the probe's surface and scans the surface of the transducer while operating in a pulse-echo mode. This testing transducer is addressed as "the transducer" to avoid confusion.

The goal of this technique is to obtain the reflections from the inner layers and provide the reflectivity data as a $2 \mathrm{D}$ image (C-scan). A wideband ( $80 \%$ at $-6 \mathrm{~dB}$ ), $20 \mathrm{MHz}$, focused transducer IRY220 (NDT transducers LLC, US) was chosen for imaging. Such bandwidth would offer a $60 \mathrm{~ns}$ resolution. A focal spot $(27 \mathrm{~mm}$ distance, $0.35 \mathrm{~mm}$ diameter, $10 \mathrm{~mm}$ length in water) was placed on the surface of the probe. The inclination angle of the focused transducer was aligned to get the maximum reflection amplitude.

First, the excitation signal was removed from the obtained waveforms. Optionally, the signals can be filtered by a bandpass filter. While the primary purpose can be the removal of the out of band noise, the high-pass filter part is useful at suppressing the low frequency oscillations caused by the excitation. Part of the waveform representing reflections from the tested probe was cut out of the filtered signal for further analysis. Yet, the signals obtained in such way are not suitable for direct gating: the scanning plane cannot be perfectly aligned to the probe's surface. The signals obtained for the probe $\mathrm{C} 2 \mathrm{P} 10 \mathrm{~N}-\mathrm{E}$ were used to produce the figures that appear in the description of the technique. They serve just as an illustration of how the technique works, see section 4.2 for the complete discussion of the transducer details and results obtained. Fig. 2 shows a typical problem found when scanning the probe surface with a high frequency transducer: the scanning plane is not perfectly parallel to the tested probe's surface so the lines representing the reflecting surface are inclined. RF signal envelope obtained by the Hilbert transform $[28,29]$ was used to produce this image.

As it is desirable to have the acquired waveforms aligned with the signal acquired at the center of the probe (reference), the following procedure is proposed. The time of flight for the front surface reflection was estimated first from the cross-correlation function (CCF) between the reference and the remaining waveforms. A rough delay (time of flight, ToF) estimation was done by locating the peak of the discrete CCF: 


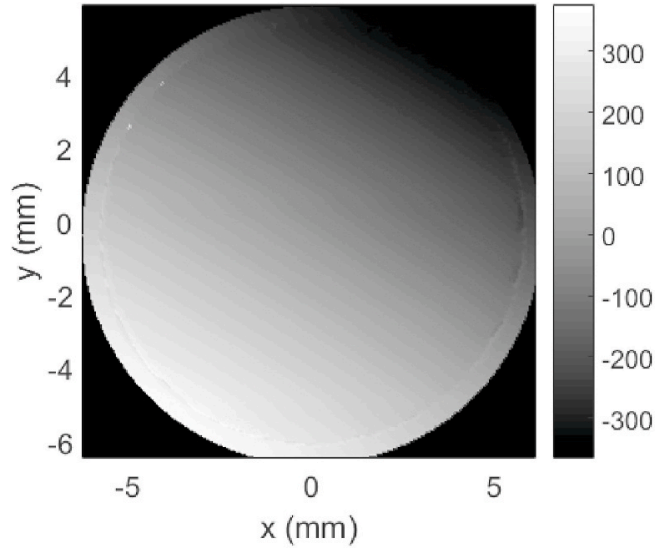

Fig. 3. ToF map (the grey scale is $\mu \mathrm{m}$, corresponding to $1500 \mathrm{~m} / \mathrm{s}$ velocity in water) with a data mask (black) visible.

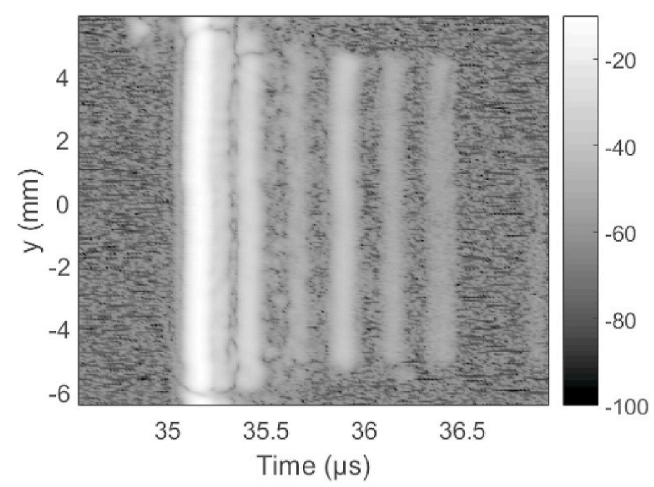

Fig. 4. An example of the aligned B-scan of the received reflections after the delay adjustment to correct the lack of parallelism between the scanning plane and the tested probe's surface (grey scale is dBV).

$m=\underset{n}{\operatorname{argmax}}\left(x_{n}\right)$,

$x_{n}=\sum_{k=1}^{N} \operatorname{sref}_{k-n} \cdot s_{k}, n=-(N-1) \ldots(N-1)$,

where $s_{\mathrm{k}}$ is any waveform and $s r f_{\mathrm{k}}$ is the reference (a waveform obtained at a probe's center), sampled at the time instances $k$, total length $N$. The actual peak usually does not reside on the sampling grid, because the CCF is discrete. Subsample offset $\delta T o F$ was estimated using the frequency domain subsample interpolation [30]. The reference signal was shifted by $m$ and the $\delta T o F$ was estimated by the weighted regression using a L2 norm $\varsigma_{\mathrm{L} 2 \mathrm{WR}}$ minimization:

$\varsigma_{L 2 W R}=\sum_{k=1}^{N}\left(\varphi\left(X_{k}^{0}\right)-\omega_{k} \cdot \delta T o F_{L 2 W R}\right)^{2} \cdot\left|S t_{k}^{0}\right|^{2}$,

where $X^{0}{ }_{k}$ is the Discrete Fourier transform of the CCF when the reference signal is shifted by $m, \omega_{\mathrm{k}}$ is the angular frequency, corresponding to a $X^{0}{ }_{k}$ component, $S t_{\mathrm{k}}$ is the Discrete Fourier transform of the reference signal.

The obtained 2D ( $\mathrm{x}$ and y coordinates) array of time shifts (ToFs) was approximated by the two-dimensional linear regression surface function:

$t_{\text {corr }}(x, y)=p_{00}+p_{01} \cdot\left(x-x_{0}\right)+p_{10} \cdot\left(y-y_{0}\right)$,

where $p_{00}, p_{01}$, and $p_{10}$ are the coefficients defining the surface elevation and inclination along $\mathrm{x}$ and $\mathrm{y}$ axis. Only the ToF values of signals

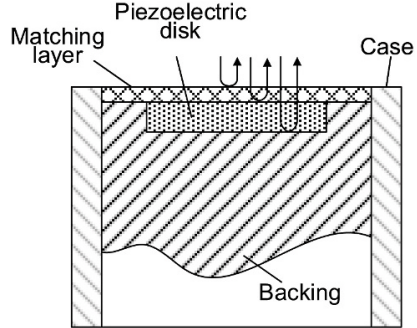

Fig. 5. A simplified ultrasonic probe's construction with the essential layers indicated.

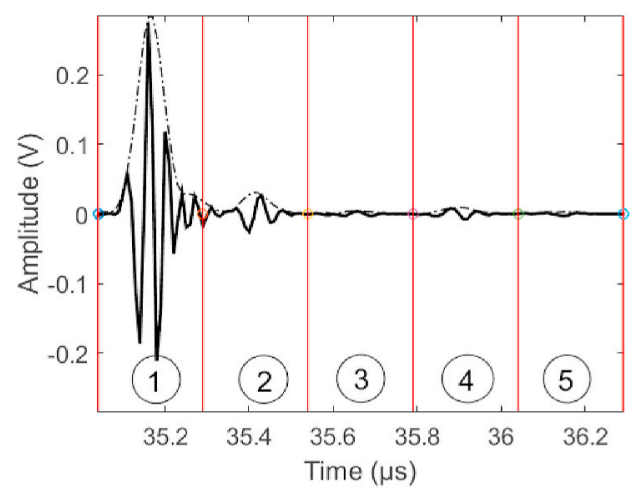

Fig. 6. An example of the reflections generated by a probe with one matching layer.

acquired at the coordinates $x, y$ within the mask (refer to a black circular vignette in Fig. 3) were used. The mask size was slightly smaller than the probe diameter and it was centered on the data obtained.

Then, all the signals $s_{\mathrm{k}}$ were aligned applying the artificial delay $t_{\text {corr }}$ by using a corresponding phase shift in the frequency domain [31]:

$s_{\text {corr }}[n, x, y]=\operatorname{IDFT}\left(\operatorname{DFT}(s[n, x, y]) \cdot e^{\omega_{m} t_{c o r r}}\right)$,

where DFT and IDFT are the direct and inverse discrete Fourier transform, respectively. Fig. 4 shows an example of the aligned B-scan of the received reflections in Fig. 2. Note, that the curvature of a front face surface remains in order to have a proper gating of the inner reflections.

The received waveform echoes were gated next. These echoes respond to the expected internal layers' stackup (presented in Fig. 5) of a probe having one matching layer, one piezoelectric layer and a backing block.

Assuming a $\lambda / 4$ thickness for the matching layer, it would take a 500 ns delay (double path, so half of the period) for a $1 \mathrm{MHz}$ probe and 100 ns for a $5 \mathrm{MHz}$ probe, respectively. For a $\lambda / 2$ piezoelectric disk thickness, it would take $1000 \mathrm{~ns}$ for a $1 \mathrm{MHz}$ probe and $200 \mathrm{~ns}$ for a $5 \mathrm{MHz}$ probe, respectively. Then, simple gating with the gates spaced at the half of the period of the center frequency can be used. If a ratio of the tested probes'

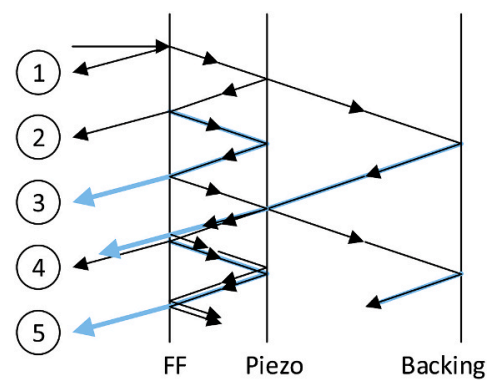

Fig. 7. A signal path analysis for the reflections observed in Fig. 6 . 


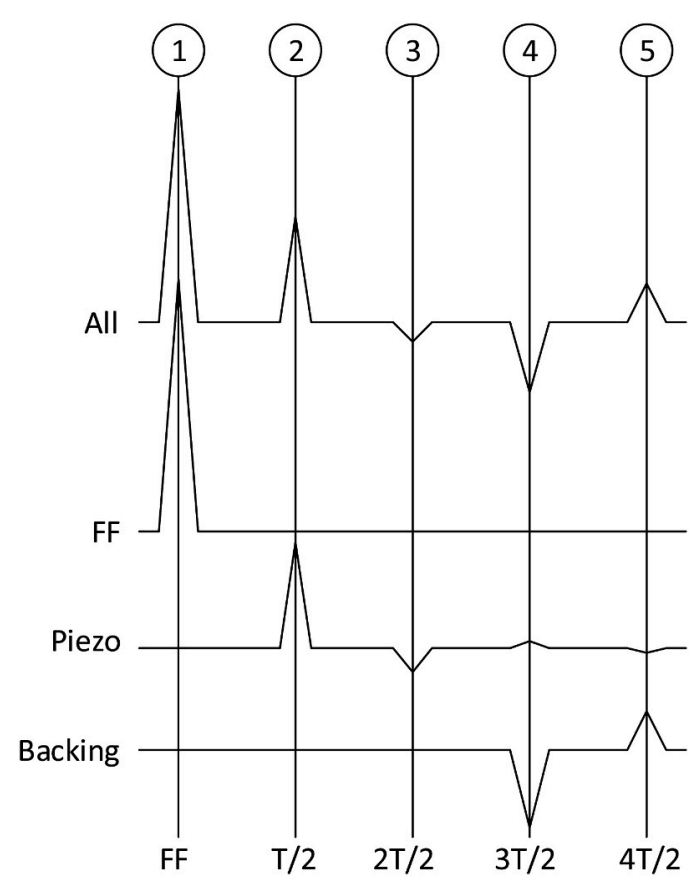

Fig. 8. Signals expected in the reflection mode for a probe with one matching layer.

and focused transducer frequencies is lower, decomposition could be a solution $[32,33]$.

An example of the waveform received by the transducer in a pulseecho mode for a $2 \mathrm{MHz}$ probe with one matching layer is presented in Fig. 6 (solid line: RF signal, dash-dot line: envelope). Red lines indicate the gate positions. The signal within the gate was used to calculate the root-mean-square (RMS) value, which was then used for the C-scan image production.

The analysis of the expected reflections is presented in Fig. 7.

The color blue in Fig. 7 indicates the polarity change. It was assumed that the matching layer and the backing block have acoustic impedances in between those of the water and the piezoelectric disk. Only the reflections occurring in the matching layer and in the piezoelectric disk are presented in Fig. 7. A temporal analysis of the reflections occurring at every interface is presented in Fig. 8.

The leftmost pulse corresponds to the front face (FF) reflection. The pulse is positive (no phase change) because a matching layer has a larger impedance (assuming $Z_{M} \sim 7.4 \mathrm{MRayl}$ ) than water ( $Z_{W}=1.5$ MRayl). The second pulse comes from the reflection at the interface between the matching layer and the piezoelement of the probe. The pulse is still positive because the piezoelement also has a larger impedance $\left(Z_{P Z T} \sim 36\right.$ MRayl) than the matching layer. The third pulse is a multiple reflection inside the matching layer, it is negative because it was reflected from water at a time instance $2(\mathrm{~T} / 2)$. The fourth pulse is prevailed by the reflection at the interface between the piezoelectric element and the backing block. It is negative, because the acoustic impedance of the backing ( $Z_{B} \sim 10$ MRayl [34]) is lower than that of the piezoelement. The amplitude of multiple reflections inside the matching layer, received at gate 4 (see Figs. 6-8) is much smaller. See Appendix A for a detailed analysis. The analysis indicates that the reflection from backing is 2.5 times larger than for multiple reflections inside the matching layer $\left(R_{\text {Lback }}=-0.18\right.$ versus $\left.R_{\text {Imatch }}=0.07\right)$.

The succeeding pulses are multiple reflections inside the matching layer and the piezoelectric layer. It can be concluded that the reflections 1,2 and 4 are of interest in case of a probe with one matching layer. Cscan of the reflection 1 can be used to confirm the stability of an incoming acoustic signal, while the reflection 2 can indicate a corrupted or delaminated matching layer. Reflection 4 can show a loss of acoustic

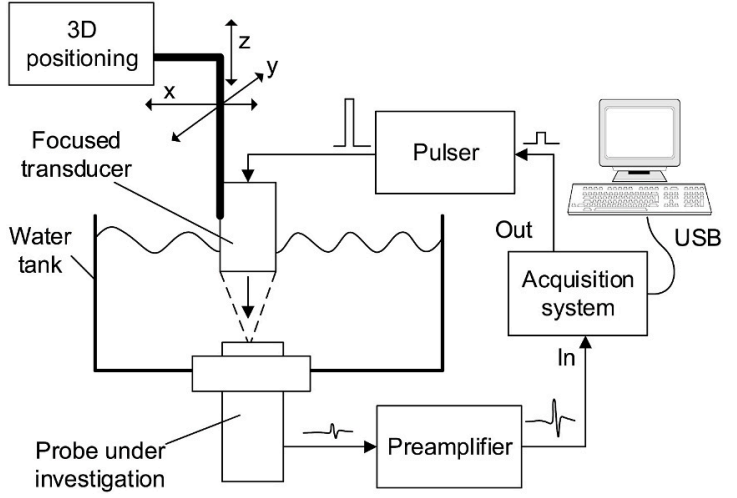

Fig. 9. Transmission imaging setup.

contact or a lack of backing material.

\section{Proposed technique: transmission imaging}

While the reflections from the probe internal structure provide a good indication of the probe's integrity features, such analysis does not fully represent all the aspects of the probe's quality. While the reflection technique can assess the integrity of the acoustic coupling and the bounding at the different interfaces in the probe, no information is obtained about the integrity of both the electrical contacts and the piezoelectric element (e.g. if it is locally damaged and the transduction capability is compromised in some areas). Moreover, no information is obtained if the acoustic layup tampers the signal propagation.

The purpose of the proposed transmission mode (see Fig. 9) is to overcome these limitations. While mechanically the setup is exactly the same as in the reflection mode (see Fig. 1), electrically it is arranged to pick up the signals transmitted from the focused transducer to the tested probe.

The same acquisition equipment as per Fig. 1 was used here. An example of the transmitted waveform for a $2 \mathrm{MHz}$ one matching layer probe is presented in Fig. 10 left (Red lines: the gate positions, solid line: the RF signal, dash-dot line: the envelope).

The received signal has energy concentrated at low frequencies due to the probe passband (refer to Fig. 10 right). The focused transducer beam width and so the spatial resolution is frequency-dependent therefore higher frequencies should be used to increase the C-scan resolution. Fortunately, a large amount of energy can still be found at high frequencies (in the passband of the focused transducer). To extract these frequencies, the received signals were filtered using a 4-th order Butterworth BP filter: the LF side suppresses the low frequencies and the HF side reduces the noise. A resulting signal has a higher center frequency (Fig. 11 right) and is more suitable for gating (Fig. 11 left).

The red vertical lines in Figs. 10 and 11 indicate the gate positions. The gates were positioned in such a way, that gate 1 takes the piezoelectric response front face signal and gate 2 takes the backwall signal. Signals within the gates were used to calculate the RMS value, which in turn was used to create the C-scan.

The analysis of the signals expected for one matching layer probe (see Fig. 7 right for signal path) is presented in Fig. 12.

It can be noted that the pulse registered by a backwall is larger than the front face (FF) signal. See Appendix B for the analysis of the cause. The sign is opposite because the front face is compressed, but the backwall is expanded for the positive polarity acoustic pulse.

The signals were aligned using the same ToF map produced for the reflection mode, in this case the ToF values were divided by two to account for the single water path in the transmission mode. Shifting was accomplished by applying the artificial delay using Eq. (5). Fig. 13 shows an example of a B-scan (envelope) obtained in the transmission mode. 

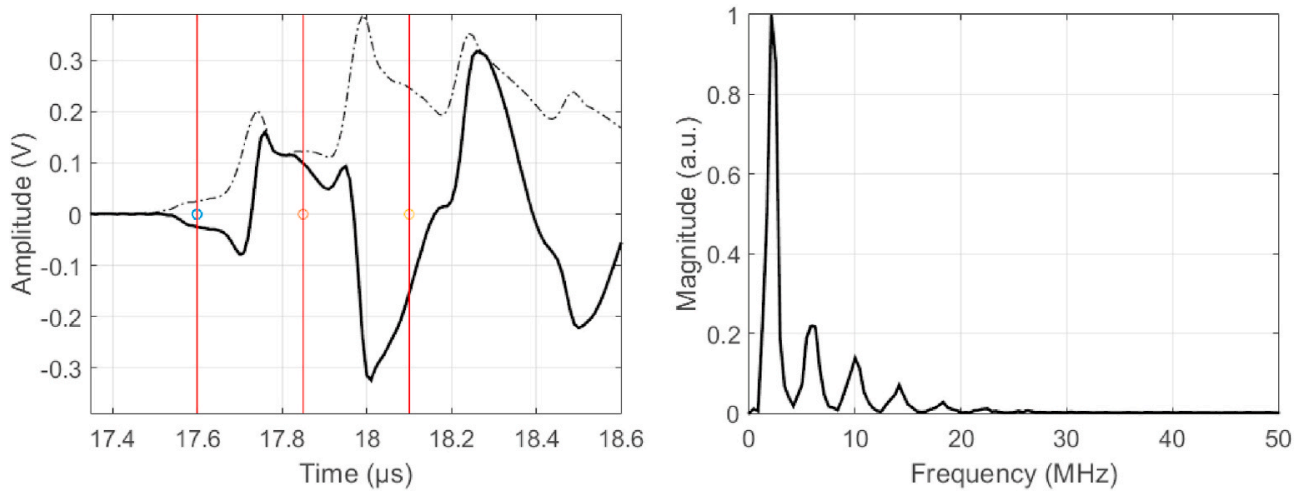

Fig. 10. The time domain (left) and the frequency domain (right) representation of the signal received at the probe's center in a transmission mode.
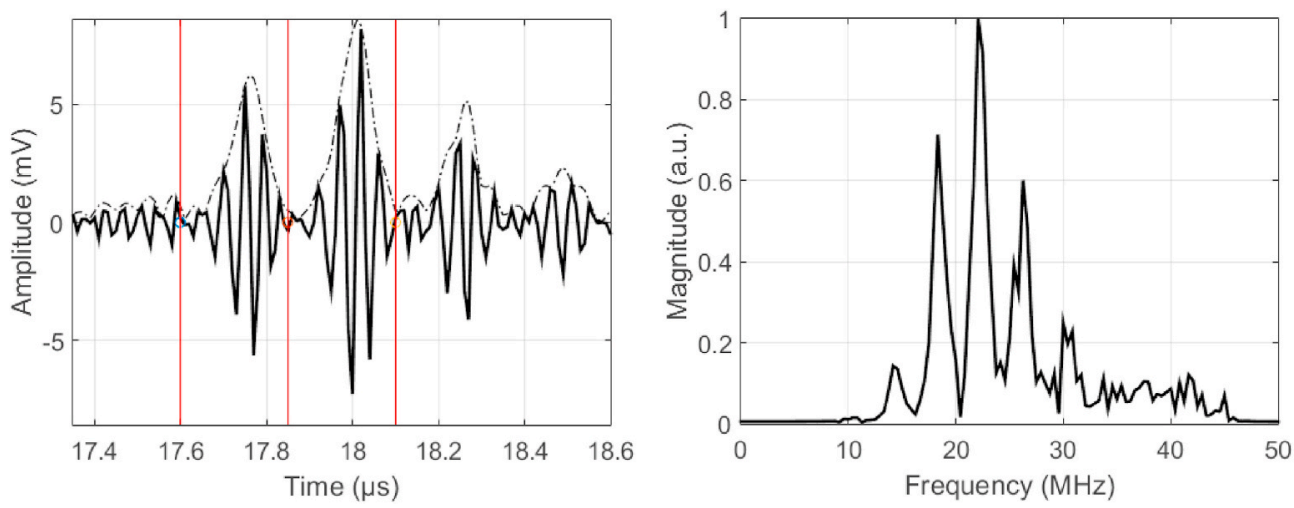

Fig. 11. A filtered (20-45 MHz BP) Fig. 10 signal in time (left) and frequency (right) domain.

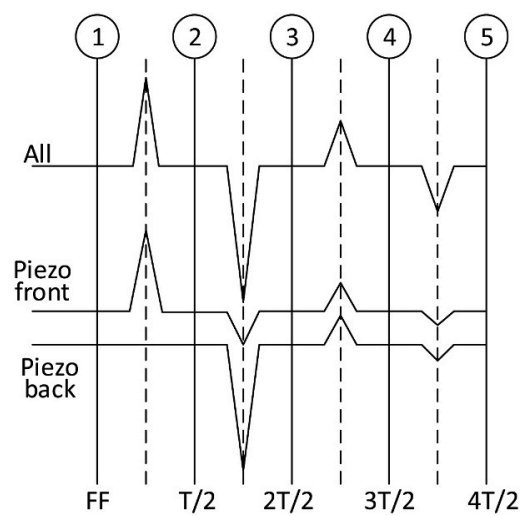

Fig. 12. Signals expected in the transmission mode at a piezoelectric element in case of one matching layer. Time scale matches Fig. 9 if an acoustic source is aligned with the FF.

The leftmost line corresponds to the electrical signal generated when an ultrasonic pulse hits the front face of the piezoelement. The incoming wave produces a positive pulse because the piezoelement is compressed (assuming positive pressure; refer Fig. 12 "Piezo front"). The next line in a B-scan corresponds to the electrical pulse generated as an ultrasound pulse hits the backwall of the disk. This wave produces a negative pulse because the piezoelement is expanded (refer Fig. 12, "Piezo back"). The succeeding lines are multiple reflections within the piezoelement.

An even simpler approach can be used: the RMS value of the filtered signal over the whole time range can be calculated to obtain the integral sensitivity that includes both a front face and a backwall. Such a measurement is less sensitive to alignment.

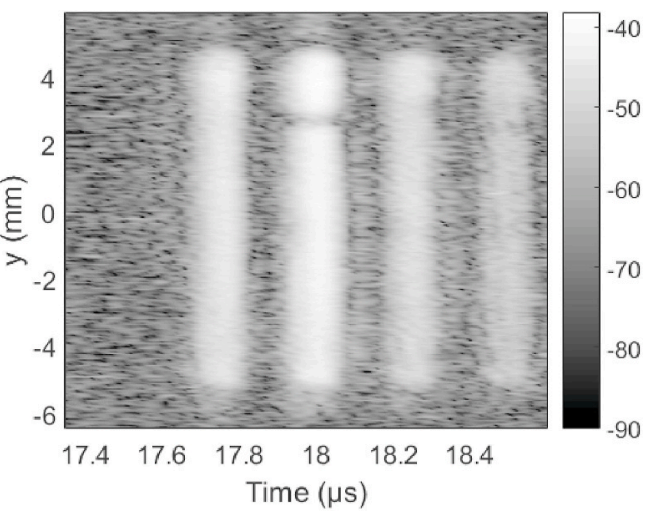

Fig. 13. B-scan of the filtered and aligned signals acquired in the transmission mode (grey scale is $\mathrm{dBV}$ ).

\section{Experimental results}

Several probe types have been investigated to test the proposed technique. A combined setup as described in Figs. 1 and 9 was used. The same focused transducer (IRY220 $20 \mathrm{MHz}$ ) was used in all the experiments. It was excited by a 20 ns rectangular $150 \mathrm{~V}$ pulse, using a transformer push-pull topology pulser of our own design. More details on the pulser can be found in Ref. [25]. In order to be able to compare the reflection and transmission test results, two acquisition channels and two reception preamplifiers were used. One channel was used for the reflection mode signal registration, as described in section 2, the other was used to register received signals in a transmission mode, as described in section 3 . The reflected and transmitted signals were 


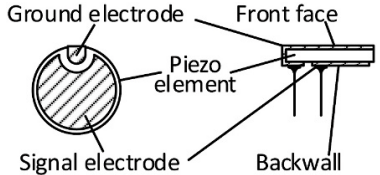

Fig. 14. Piezoelectric disk used for investigation: back (left) and side (right) view projections with the soldered wires attachment shown.

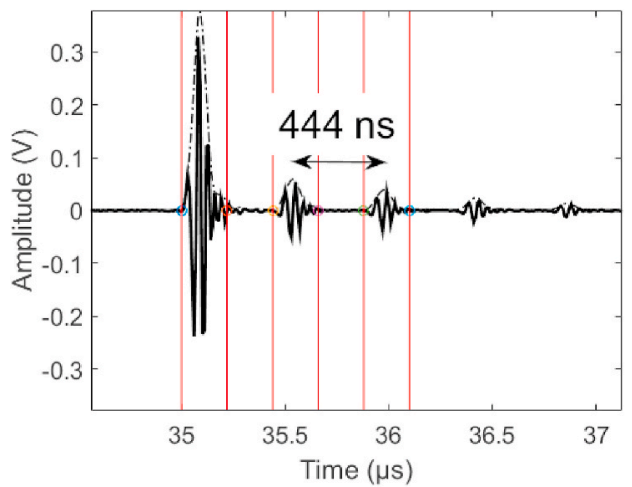

Fig. 15. Waveform obtained at the center of the disk $(x=0 \mathrm{~mm}, y=0 \mathrm{~mm})$ in the reflection mode for a bare piezoelectric disk at the center of the disk. Solid line: RF signal, dash-dot line: envelope.

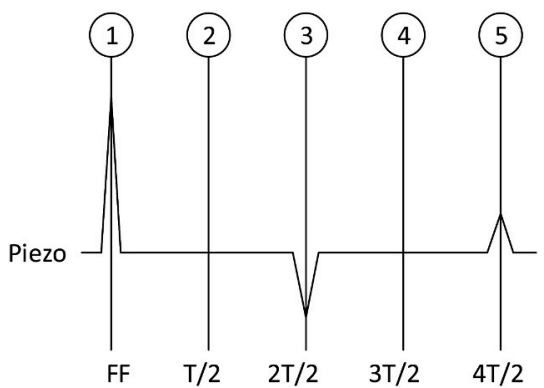

Fig. 16. Signals expected in the reflection mode for a bare piezoelectric disk.

amplified using a PA1008 preamplifier (AUT Solutions, US, TX). The preamplifier had a high voltage protection circuit, $1 \mathrm{k} \Omega$ input impedance [26]. An acquisition system of our own design [27] was used for data acquisition. It has two 10 bit ADC channels operating at $100 \mathrm{MHz}$, therefore the reflection and transmission mode signals were acquired simultaneously. The amplified and sampled signals were transferred to a host PC using a high speed USB 2.0 interface [27]. The preamplifiers' gains were adjusted $(0-60 \mathrm{~dB})$ for every probe individually to match the acquisition system's dynamic range. The scanning area was selected according to the tested probe's size.

\subsection{Simple piezoelectric disk}

A bare PZT ceramics disk immersed in water was first investigated as the simplest probe. The disk diameter was $10 \mathrm{~mm}$ and the thickness was $1 \mathrm{~mm}$. The disk is provided with a ground electrode that wraps around the disk (see Fig. 14) for the ground connection attachment. Such a ground electrode structure creates an artificial insensitivity zone: the area where the ground electrode wraps the piezoelement is not sensitive for the incoming pressure.

The signals were collected by scanning a $10 \times 10 \mathrm{~mm}^{2}$ area with a $0.05 \mathrm{~mm}$ step (a set of $200 \times 200$ waveforms was collected). The gain was set to $16 \mathrm{~dB}$ in the reflection mode and it was $21 \mathrm{~dB}$ in the transmission mode.

The signal obtained in the reflection mode is shown in Fig. 15. The reflection mode signals were not filtered. This waveform matches the prediction of the analysis presented below (see Fig. 16).

Reflections expected in this case are analyzed in Fig. 16.

The first pulse corresponds to the front face (FF) reflection. It is positive because the piezoelement's impedance is higher than water's. The next pulse is the reflection from the backwall of the piezoelement (disk). It is negative because the piezoelement has a higher impedance than water. The next pulses are multiple reflections within the piezoelement. While the center frequency specified by the manufacturer was $2 \mathrm{MHz}$, the investigation revealed that the piezoelement propagation time corresponds to $2.25 \mathrm{MHz}$ (444 ns according to Fig. 15). The gates' positions were adjusted accordingly.

A B-scan (Fig. 17 left) over the area where the disk is tampered by the ground electrode wrapping reveals that the backwall reflection is affected by the soldering of the wire (see Fig. 14 right), so reflections from the backwall are distorted (upper part of the image in Fig. 17 left).

The first gate ( 1 according to Fig. 16) represents the reflection from the disk front face (Fig. 17 right). The rim, created by the upper electrode, can be seen. No defects on the front face are visible.

The second gate ( 2 according to Fig. 16) is set for the reflection inside the disk, between the front face and the backwall (Fig. 18 left). A small part of the disk was chipped at the backwall. A bright spot at the leftmost middle part of the image indicates this chip-off.

The third gate (3 according to Fig. 16) represents the disk backwall reflection (Fig. 18 right). Ceramics chip-off spot at the leftmost middle part of the image is visible better now. Also, the ground electrode metallization layer and the wire soldering points can be seen (upper middle part of the image).

The signals received in the transmission mode were filtered using a
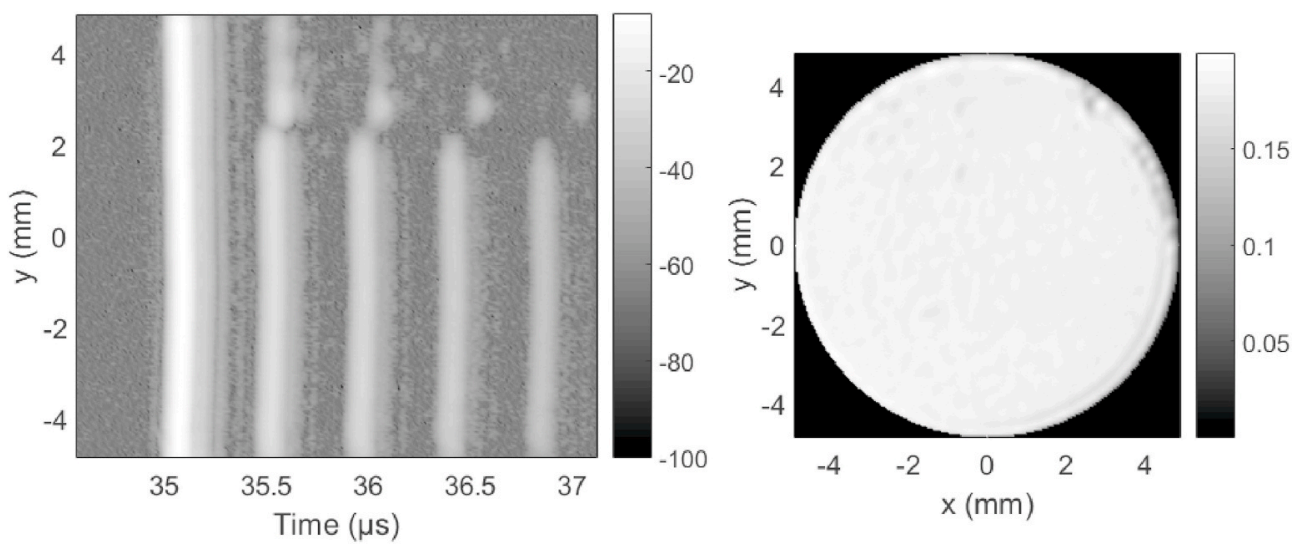

Fig. 17. B-scan at the disk center (left, grey scale is dBV) and C-scan at the front face (right, grey scale is V) in a reflection mode. 

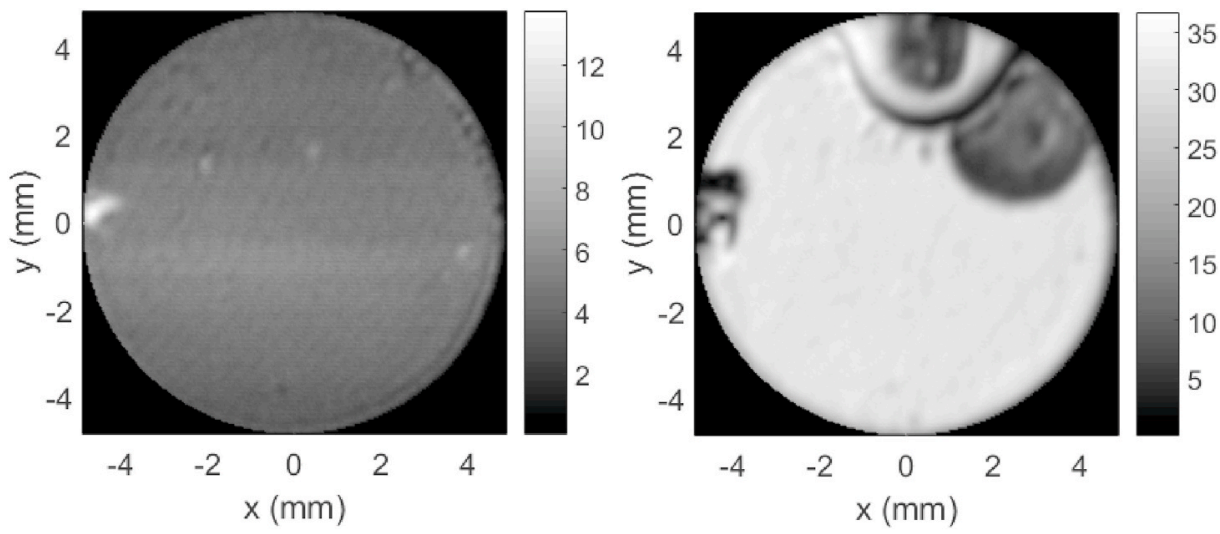

Fig. 18. Piezoelectric disk C-scans in the reflection mode (grey scale is mV): inside of the disk (left) and at the backwall (right).

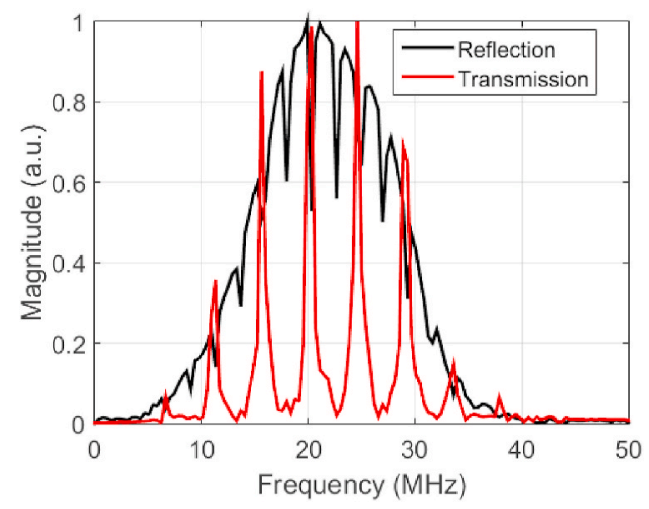

Fig. 19. Spectrum of the signals obtained from a bare piezoelectric disk at the center of the disk $(x=0 \mathrm{~mm}, y=0 \mathrm{~mm})$ in a reflection mode (black line) and a transmission mode (red line). (For interpretation of the references to color in this figure legend, the reader is referred to the Web version of this article.)

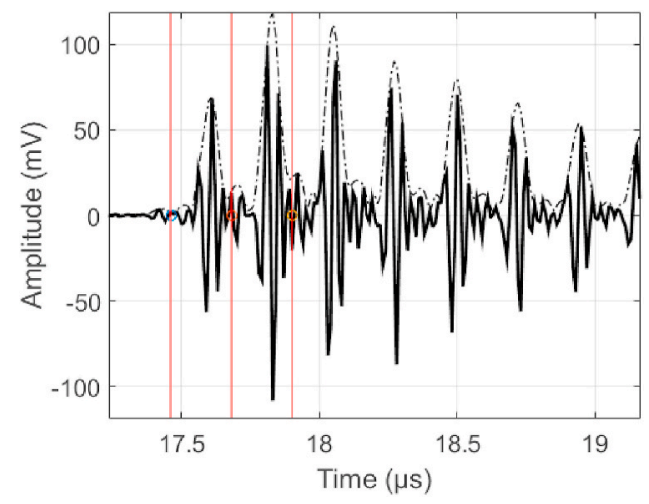

Fig. 20. Waveform obtained in the transmission mode at the center of the disk.

$17 \mathrm{MHz}-45 \mathrm{MHz}$ 4-th order Butterworth BP filter. The filter passband was adjusted to match the spectrum envelope of the reflection and transmission mode signals (Fig. 19).

Characteristic peaks, corresponding to odd harmonics of $2.25 \mathrm{MHz}$ fundamental frequency can be noted in a transmission mode signal spectrum. Spectral dips, corresponding to multiple reflections within the disk can be seen on a reflection mode signal spectrum.

The filtered transmission mode signal registered at a disk center is presented in Fig. 20.

The transmission mode signal analysis based on Fig. 21 is presented below.

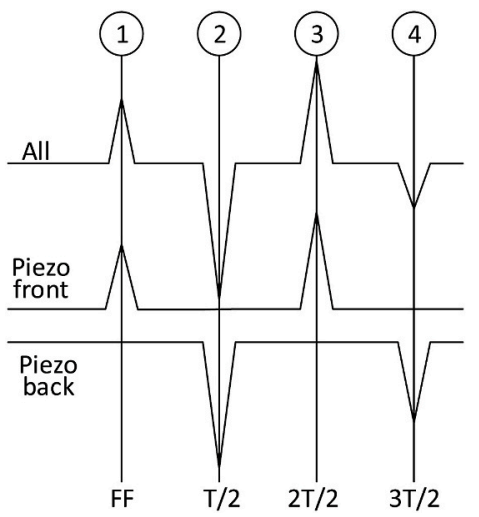

Fig. 21. Signals expected in the transmission mode for a bare piezoelectric disk.

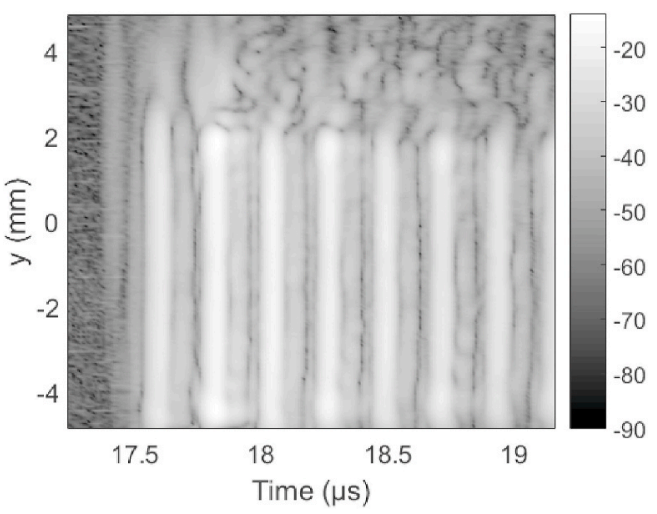

Fig. 22. Piezoelectric disk B-scan in the transmission mode at the center (grey scale is $\mathrm{dBV}$ ).

Again, as explained earlier, the pulse registered by the backwall is larger than the FF signal (Fig. 21). The same can be confirmed by looking at the actual signal (Fig. 20).

A slice B-scan (Fig. 22) over the area where the disk is tampered by the ground electrode wrapping reveals that there is no signal registered at the area where the ground electrode is on both sides of the piezoelement (upper part of the image).

It can be noted that the front face has a slightly narrower insensitive area (leftmost line, upper part) due to the lesser influence of the electrode tampering (on the backwall). C-scan obtained when the RMS value of the whole signal is taken (Fig. 23) represents the integral sensitivity of 

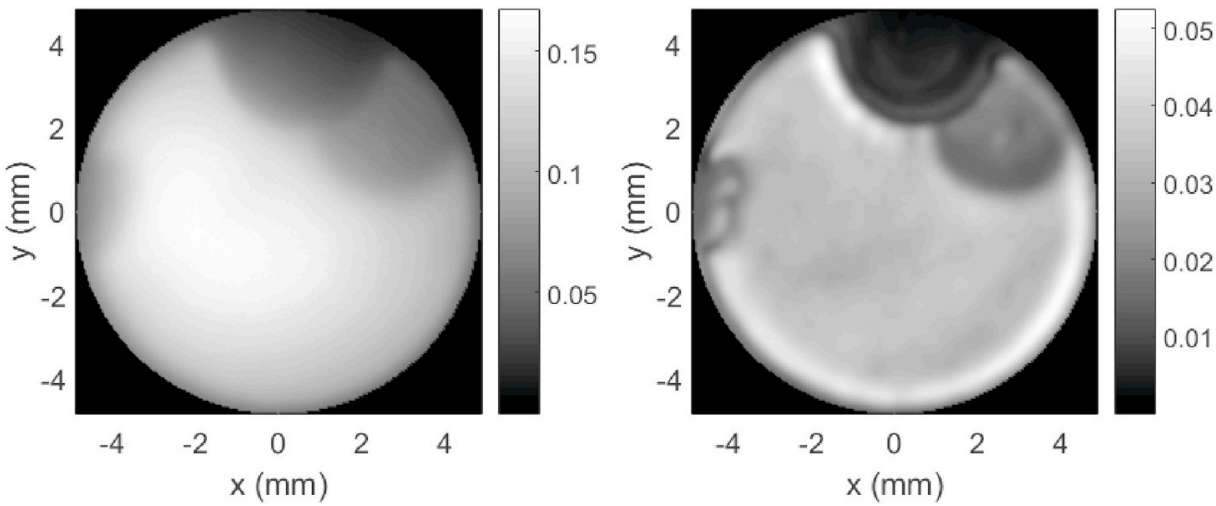

Fig. 23. Piezoelectric disk C-scan of the whole signal in the transmission mode (grey scale is mV): unfiltered (left) vs. $17 \mathrm{MHz}-45 \mathrm{MHz}$ PB filtered (right).
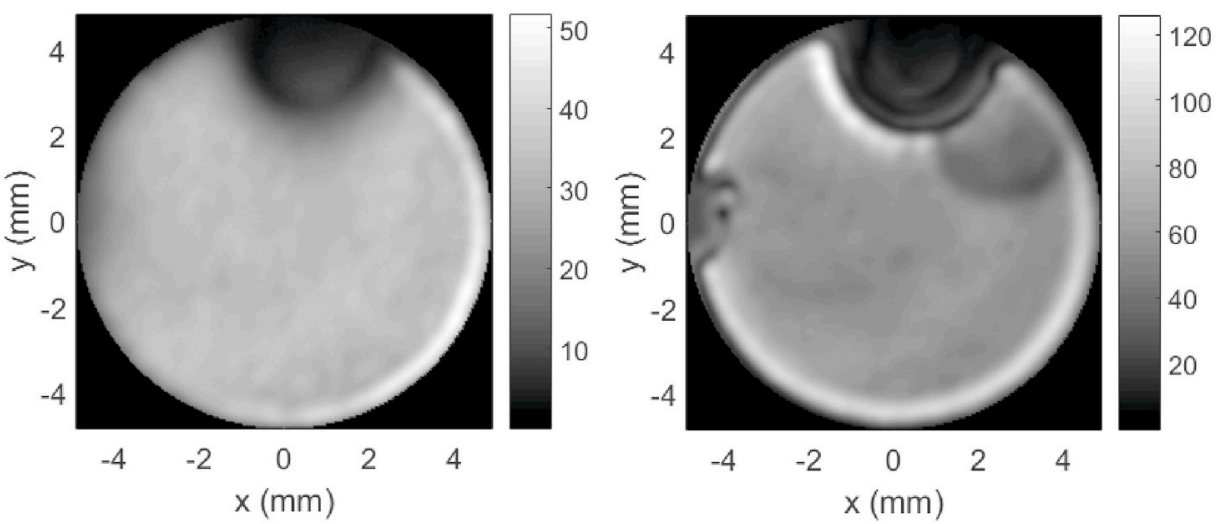

Fig. 24. Piezoelectric disk C-scan in the transmission mode (grey scale is mV): front face (left) and backwall (right).

the disk.

The dark spot at the upper part of the image is due to the tampered electrode. The essential advantage of the transmission mode is demonstrated here: the reflection imaging does not reveal the sensitivity loss (where the ground electrode wraps around the disk, refer to Fig. 14) while the transmission imaging clearly indicates the areas of sensitivity loss. It can be concluded that the resolution is low if an unfiltered signal is used for the sensitivity imaging. Moving the passband to the upper frequencies increases spatial resolution of the C-scan obtained. Yet, this comes at the expense of the reduced signal-to-noise ratio: higher frequency images exhibit more grain noise. PB filtered (17 MHz-45 MHz) signals were used in further analysis.

The first gate ( 1 according to Fig. 21 ) represents the sensitivity of the disk front face (Fig. 24 left, filtered). It can be confirmed that there is no sensitivity in the area tampered by the ground electrode. However, the sensitivity loss is gradual due to the distant tampering electrode, therefore the border is blurred.

Meanwhile, the second gate (2 according to Fig. 21) reveals a different situation for the backwall sensitivity (Fig. 24 right). The sensitivity image is sharp in this case: the area of a tampering electrode is clearly visible. The area, where the piezoelectric disk was damaged (a chip-off at the leftmost middle part of the image) indicates a significant sensitivity loss at a backwall. The front face image does not indicate sensitivity loss at a chip-off area, because the ceramics part was intact at the front face.

The signals obtained in the reflection and transmission modes can be compared if an acoustic source is artificially matched to the front face. The time axes for the reflection mode $\left(t_{T X 0}\right)$ and for the transmission mode $\left(t_{R \times 0}\right)$ signals plotting were generated as follows:

$t_{T X 0}=T_{s}\left(n-n_{\text {offset }}-n_{0}\right)$,

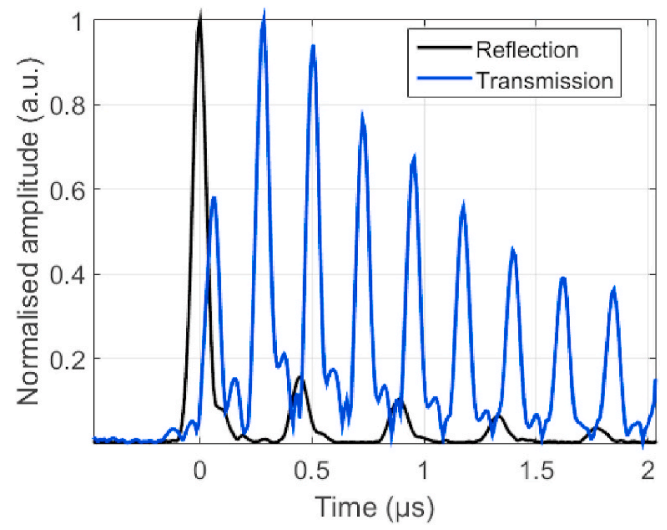

Fig. 25. A comparison of a signal envelope in the reflection (black) and transmission (blue line) modes for a bare piezoelectric disk. (For interpretation of the references to color in this figure legend, the reader is referred to the Web version of this article.)

$t_{R X 0}=T_{s}\left(n-n_{\text {offset }}-n_{0} / 2\right)$

where $T_{s}$ is a sampling period, $n$-sample number ( 0 to $N$ ), starting from the acquisition trigger, $n_{\text {offset }}$ is the delay (in samples) of an excitation signal starting from the acquisition trigger and $n_{0}$ - position of the first reflection (from the front face). The reflection peak position was used as $n_{0}$. This corresponds to shifting both signals in time, accounting for the double propagation path. The envelope plot of normalized Figs. 15 and 20 signals with the time axes transformed using (10) and (11) is presented in Fig. 25. 


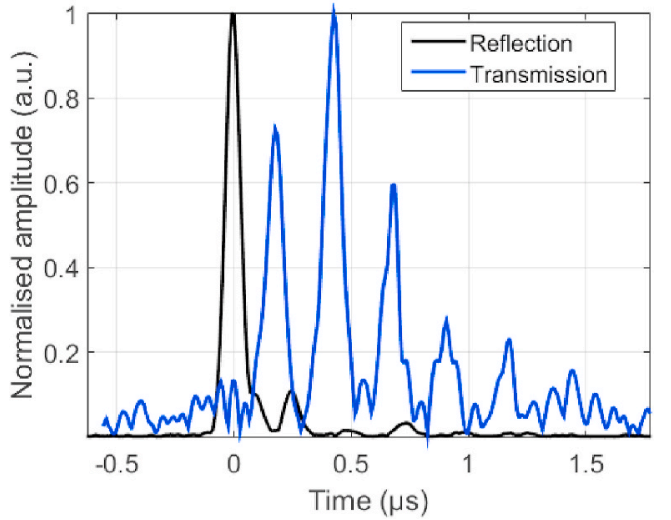

Fig. 26. A comparison of the reflection and transmission mode signal envelopes for the C2P10N-E probe.

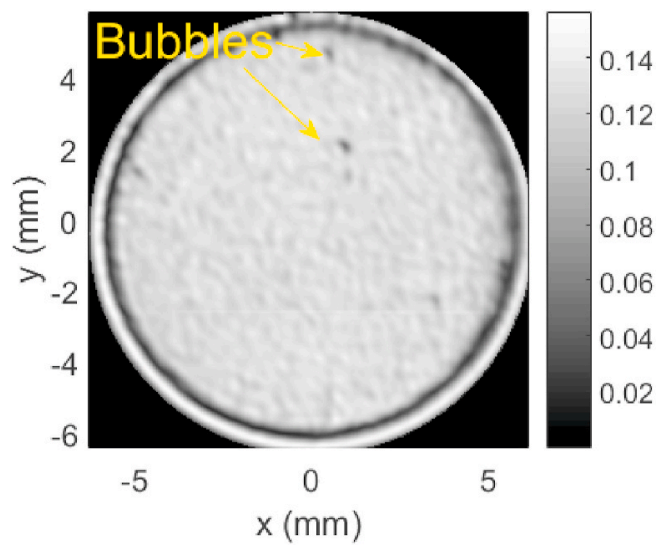

Fig. 27. Front face reflection in the reflection mode for the C2P10N-E probe (grey scale is V).

It can be seen that both the transmission mode pulse from the front face and the reflection mode pulse from the front face appear at same time.

This experiment confirmed that the sensitivity loss can be detected using only the transmission mode.

\subsection{Intact probe with one matching layer}

The probe C2P10N-E (from Doppler Electronic Technologies Inc.) was then investigated. This case can be considered as an example of a probe having a one matching layer. This is a $2 \mathrm{MHz}$, unfocused, contact probe, and has a $10 \mathrm{~mm}$ diameter piezoelement. As this probe has been barely used, no sensitivity degradation was noted.

Signals were collected by scanning a $12.5 \times 12.5 \mathrm{~mm}^{2}$ area with a $0.05 \mathrm{~mm}$ step (a $250 \times 250$ waveform set was collected). The reflection mode reception gain was $13.4 \mathrm{~dB}$, the transmission mode reception gain was $22.5 \mathrm{~dB}$.

A comparison of the signals obtained in the reflection and transmission mode with the aligned time axes (the acoustic source is artificially moved to the front face) is presented in Fig. 26.

It can be seen that the first transmission peak occurs between the reflections from the front and the backwall faces. This matches well with the predictions in Figs. 8 and 12 made for the single matching layer case.

The probe front face reflection (gate 1 according to Fig. 8) is presented in Fig. 27.

A few dark spots are caused by the small air bubbles accumulated on the front face during scanning. The rim around is created by the reflection from the probe's metal case.

The piezoelement reflection (gate 2 according to Fig. 8 ) is presented in Fig. 28 left. Same dark spots are visible where air bubbles deflected the ultrasonic waves from penetrating into the probe's inner layers. The bubble shades are slightly blurred. The dark artefact visible on the rightmost middle part is probably due to some defects in either the matching layer or the ground wire attachment.

Reflection from the backing (piezoelement backwall, gate 4 according to Fig. 8) is presented in Fig. 28 right. Same dark spots are visible where the air bubbles were located. An (now bright) artefact visible on the rightmost middle part is probably from some defect in the matching layer which increased the signal coupling. An additional dark spot appears on the upper left corner. These could be wire solder points.

The signals received in the transmission mode were filtered using a $20 \mathrm{MHz}-45 \mathrm{MHz}$ 4-th order Butterworth BP filter. Refer to Fig. 29 left for the front face sensitivity C-scan image. Note, that only defects appearing in a signal propagation path before the front face are visible: dark spots from air bubbles on front face and bright spot on the rightmost middle part, which can be attributed to a ground wire soldering point.

Fig. 29 right shows the backwall sensitivity image. It can be noted, that both defects appearing in a signal propagation path before the front face and backwall-associated defects are visible: dark spots from air bubbles on front face; dark spot, that we associated with signal wire soldering (left upper middle part in Fig. 28 right) is not pronounced as much, indicating that sensitivity is not lost here. A new defect was revealed: a large dark spot (blue arrow at middle top), indicating the sensitivity loss, which was not present in all previous images.

\subsection{Damaged probe with one matching layer}

Probe TF2C6N (from Doppler Electronic Technologies Inc.) was
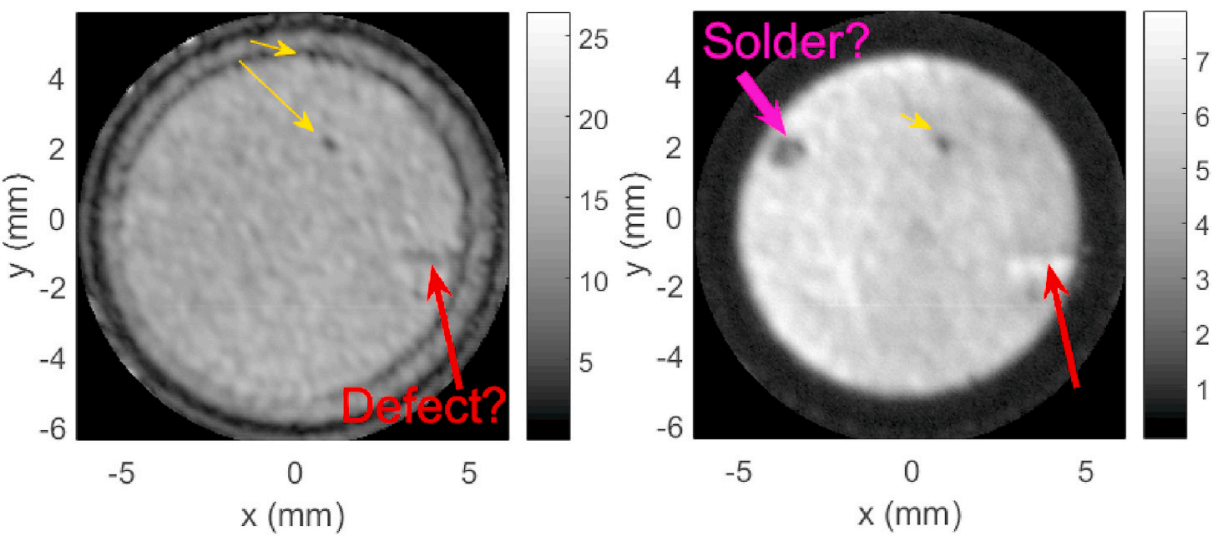

Fig. 28. C-scan of the piezoelement (left) and backing (right) reflections for the C2P10N-E probe (grey scale is mV). 

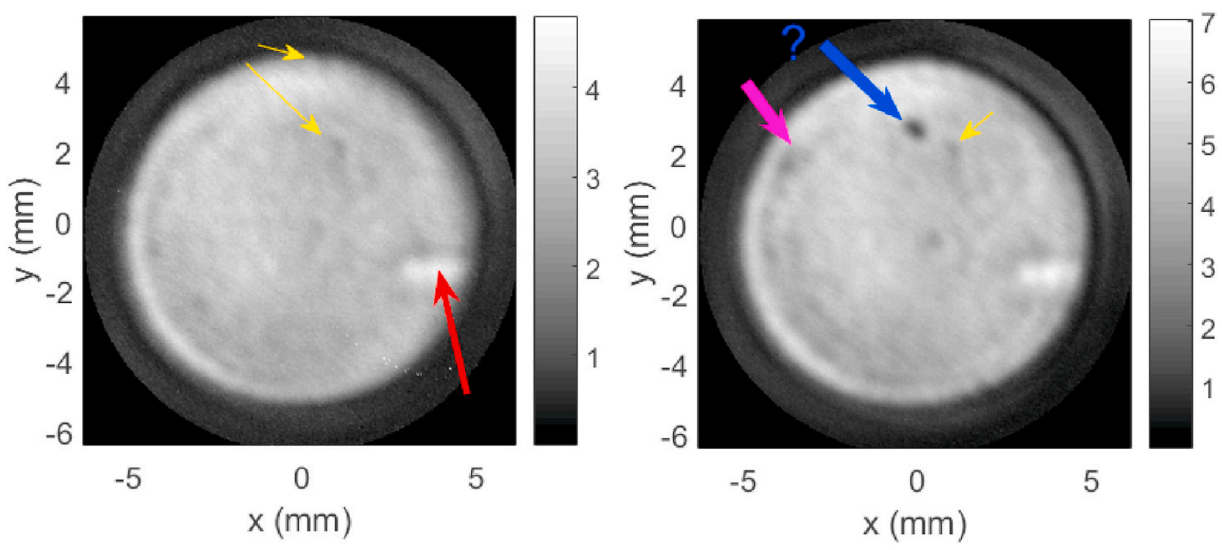

Fig. 29. A comparison of the front face (left) and backwall (right) sensitivity for the C2P10N-E probe obtained in the transmission mode probe (grey scale is mV).

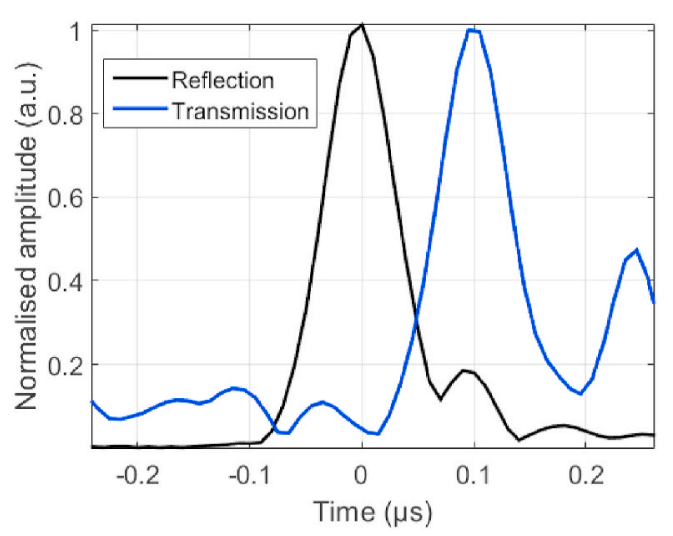

Fig. 30. A comparison of the reflection mode and transmission mode signals envelopes for the TF2C6N probe.

investigated as another representative of the one matching layer structure. This is a $2 \mathrm{MHz}$, unfocused, contact probe (used with a delay line or a wedge), and has a $6 \mathrm{~mm}$ diameter composite piezoelement. This probe has been intensely used, and a sensitivity drop has been reported (from manufacturer specified $-51.4 \mathrm{~dB}$ to $-44.69 \mathrm{~dB}$, measured according to chapter 7.4 of [1], using a $20 \mathrm{~mm}$ Plexiglass block), however, a visual inspection of the probe surface revealed no defect. The purpose then was to verify if the proposed technique can provide an explanation for this sensitivity reduction and if it can reveal any defect responsible for this.

The signals were collected by scanning $10 \times 10 \mathrm{~mm}^{2}$ area with a $0.05 \mathrm{~mm}$ step $(200 \times 200$ waveform set was collected $)$. The gain was set to $12 \mathrm{~dB}$ and $20 \mathrm{~dB}$ in the reflection and transmission modes, respectively. Reflection mode signals were not filtered. Transmission mode signals were filtered using a 15-35 $\mathrm{MHz}$ BP filter.

A comparison of the reflection and transmission modes signals (at $x$ $=-2.1 \mathrm{~mm}, y=-0.8 \mathrm{~mm}$ ) with aligned time axis is presented in Fig. 30 .

It can be concluded that the front layer is very thin in this probe: the first transmission peak occurs immediately after the front face reflection and is offset by $50 \mathrm{~ns}$, not $125 \mathrm{~ns}$ and the second reflection is delayed by $100 \mathrm{~ns}$, not $250 \mathrm{~ns}$ (which would correspond to a $\lambda / 4$ matching layer thickness).

The analysis of the probe front face reflection (Fig. 31 left) did not reveal any damage. Quite on the contrary, reflection from the piezoelement (Fig. 31 right) clearly revealed a defect that can be interpreted as a delamination at a probe's center.

The signals taken at the intact position (Fig. 32, refer black crosshair at $x=-2.1 \mathrm{~mm}, y=-0.8 \mathrm{~mm}$ in Fig. 31) and at the delamination position (Fig. 33, refer white crosshair at $x=1.25 \mathrm{~mm}, y=0.75 \mathrm{~mm}$ in Fig. 31) confirm the finding.

It should be noted, that the transmission mode signal has to be filtered using a BP filter, so much less energy remains (compare vertical axis for the left and right figures: the amplitude reduction is almost by two orders of magnitude). A large portion of the signal is concentrated at the passband of the tested probe $(2 \mathrm{MHz}$ ), therefore more noise is present in the transmission signal after the extraction of the high frequency components.

Ringing, produced by delamination, is so strong that it masks the absence of reflection from the backing: the gate, representing the reflection from backing (Fig. 34) does not contain a dark spot where delamination is present (compare with Fig. 31 right).

The transmission mode clearly demonstrates the sensitivity loss: both the whole received signal (Fig. 35 left) and the front face signal (Fig. 35 right) indicate the sensitivity loss at a probe center.
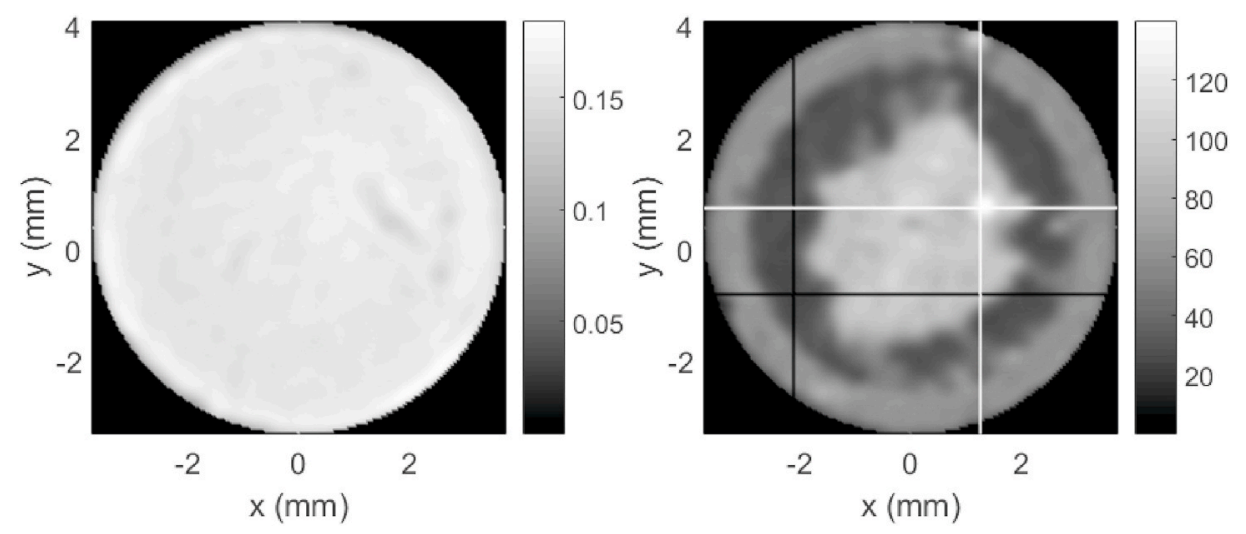

Fig. 31. C-scan of the front face (left, grey scale is V) and the piezoelement (right, grey scale is $\mathrm{mV}$ ) in the reflection mode for the TF2C6N probe. 

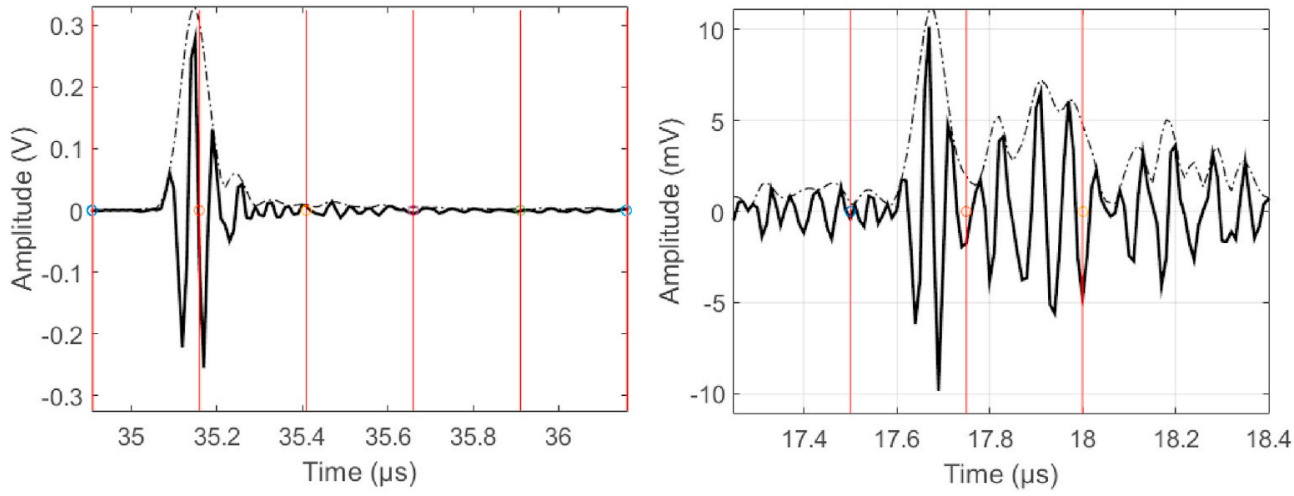

Fig. 32. A-scan for the intact position (at $x=-2.1 \mathrm{~mm}, y=-0.8 \mathrm{~mm}$, black crosshair in Fig. 31) in the reflection mode (left) and the transmission mode (right) for the TF2 6 N probe.
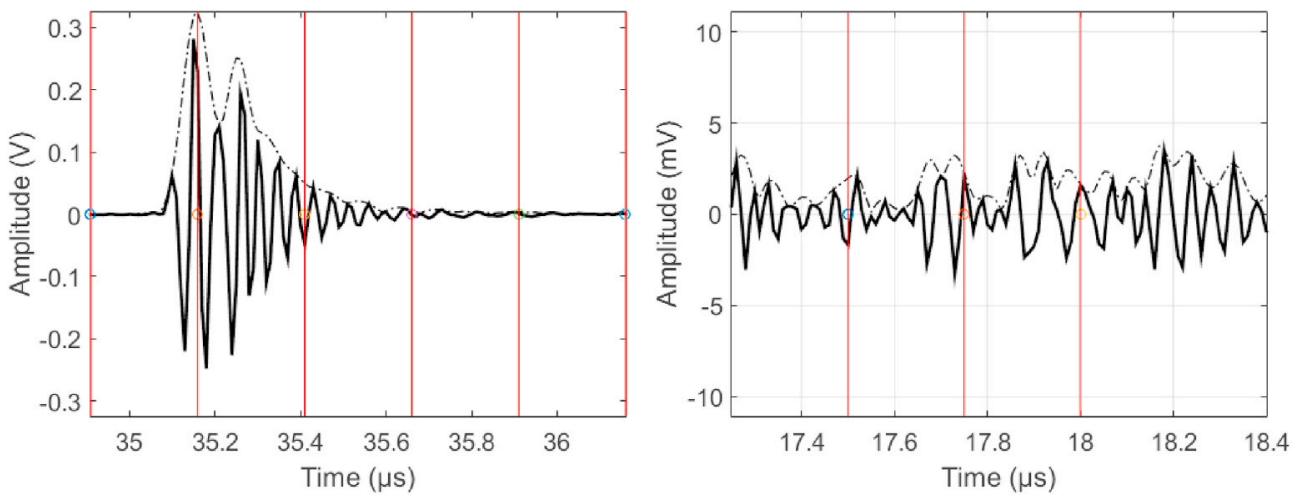

Fig. 33. A-scan for delamination (at $x=1.25 \mathrm{~mm}, y=0.75 \mathrm{~mm}$, white crosshair in Fig. 31) in the reflection mode (left) and the transmission mode (right) for the TF2C6N probe.

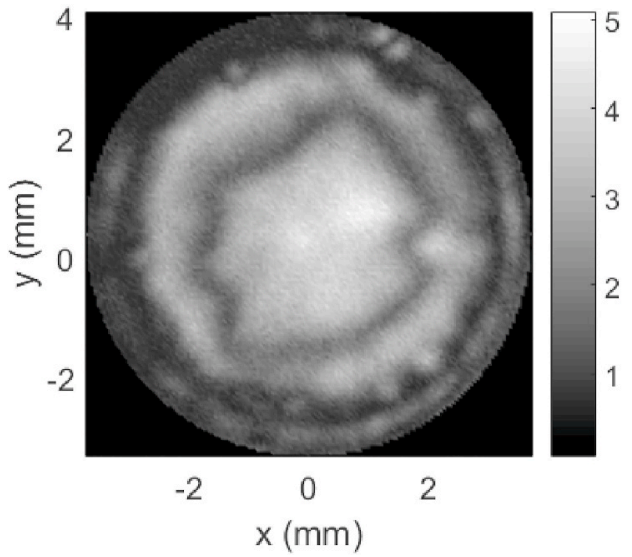

Fig. 34. C-scan of the reflection from backing for the TF2C6N probe (grey scale is $\mathrm{mV}$ ).

It can be concluded that the transmission mode is a convenient and easy tool for a spatial sensitivity evaluation and can be used as a probe's integrity measure.

\subsection{Probe with two matching layers}

The probe C543-SM (from Olympus Corp.) was investigated as an example of a probe with two matching layers. This is a $5 \mathrm{MHz}$, unfocused, contact probe, and has a $6 \mathrm{~mm}$ diameter composite piezoelement. The probe was rarely used, so no sensitivity degradation was noted.
The signals were collected by scanning $10 \times 10 \mathrm{~mm}^{2}$ area with a $0.05 \mathrm{~mm}$ step in the reflection and transmission modes simultaneously. The transmission mode signals were filtered using a $25-45 \mathrm{MHz} \mathrm{BP}$ filter.

The comparison of the reflection and transmission mode signals with the aligned time axis is presented in Fig. 36.

It can be concluded that there are two matching layers: the first transmission peak is offset by $100 \mathrm{~ns}$, which would correspond to half period delay of a $2 \times \lambda / 4$ matching layer thickness. The second transmission peak is lagging the first by $100 \mathrm{~ns}$, which corresponds to a $\lambda / 2$ piezoelectric disk thickness. See Fig. 37, left, for the expected reflection mode signals and Fig. 37 right for the expected transmission mode signals. The time scale matches if an acoustic source was aligned with the FF.

It can be concluded that the reflections $1,2,3$ and 5 are of interest in the case of two matching layers. The reflections from the front face ( 1 in Fig. 37 left) are presented in Fig. 38 left and the second matching layer reflections ( 2 in Fig. 37 left) are presented in Fig. 38 right.

An artefact seen across a matching layer C-scan (an almost horizontal dark line in Fig. 38 right) is coming from some discontinuity implemented at a first matching layer.

Reflections from a piezoelement (3 in Fig. 37 left) are presented in Fig. 39 left and reflections from backing ( 5 in Fig. 37 left) are presented in Fig. 39 right.

A shadow from discontinuity implemented in the first matching layer can be seen on both images. Two lines at $45^{\circ}$ slope, that are visible on both images, are probably due to the ground wire, run on the top of the piezoelement.

The transmission mode signals registered from the piezoelement are presented in Fig. 40: left is for front face (2 in Fig. 37 right) and right - 

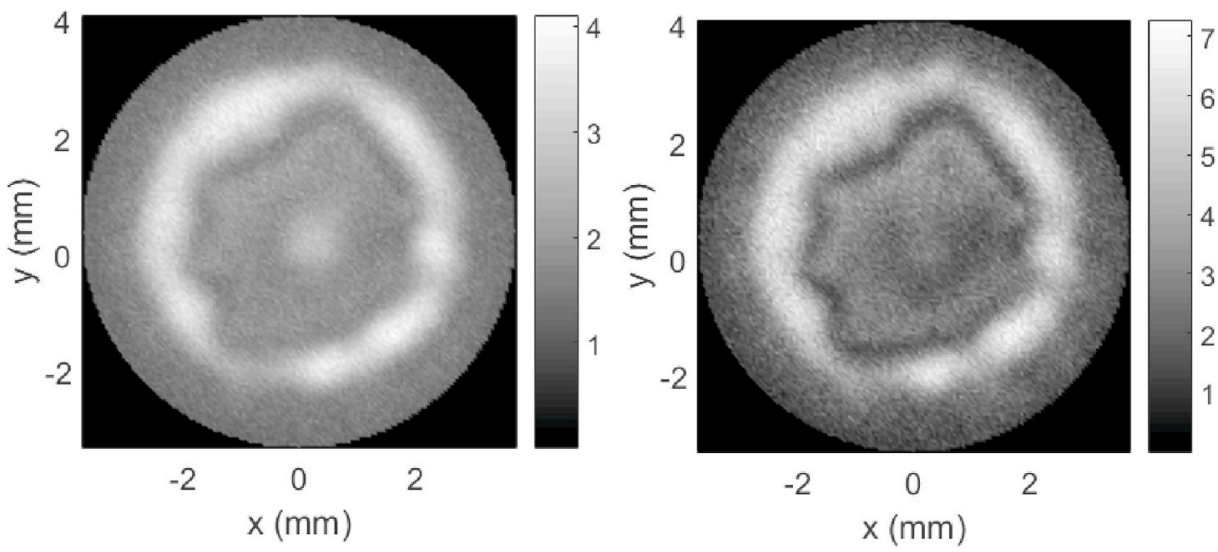

Fig. 35. C-scan of the whole signal (left) and the gated at front face (right) in the transmission mode for the TF2C6N probe (grey scale is mV).

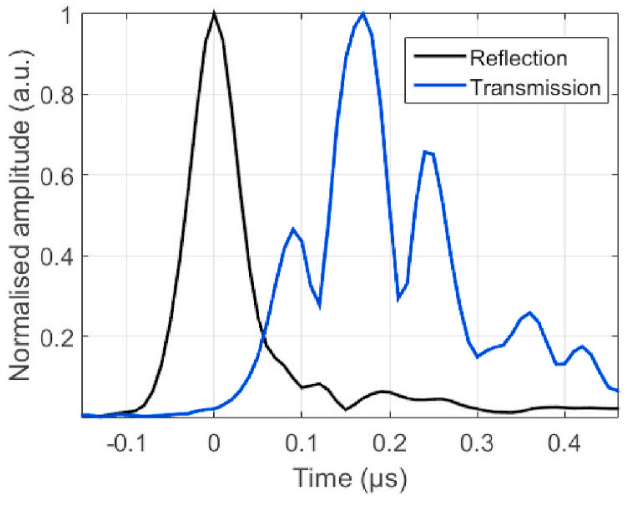

Fig. 36. A comparison of the reflection mode and transmission mode signal envelopes for the C543-SM probe.

for backwall (3 in Fig. 37 right).

It can be seen that the artefacts found in the reflection mode are also present here. The sensitivity loss is minimal where the artefacts are located. An investigation confirmed that the transducer integrity was not lost.

\section{Conclusion}

A technique for the probe's integrity testing has been proposed and experimentally verified. A reflection mode can be used in determining where a delamination has occurred. This mode requires more processing (signals have to be aligned, gates' positions derived) and provides the delamination position. Yet, there might be other defects present, like the loss of an electrode, porosity. The proposed transmission mode is suitable for a two dimensional sensitivity assessment, when aforementioned defects can be revealed. The technique is attractive thanks to its simplicity: only a focused transducer and a scanner are required to collect the data; a simple filtering is enough to obtain the transmission mode signals which are later submitted to the RMS value calculation. Only the energy of received signals can be used for imaging. If the imaging is augmented by the gating of the received signal, the influence of defects in front-matching and backing on sensitivity can be separated.

The technique can be used in several applications: i) to check the integrity of a probe already in use and verify that it is still working properly; ii) to verify the fabrication quality and confirm that all layers are properly manufactured and glued; iii) to aid in the development of the new fabrication routes, where one can check the adherence, compatibility, homogeneity, etc. of new materials or new fabrication technologies.

The data collected during the experiments are available at Mendeley
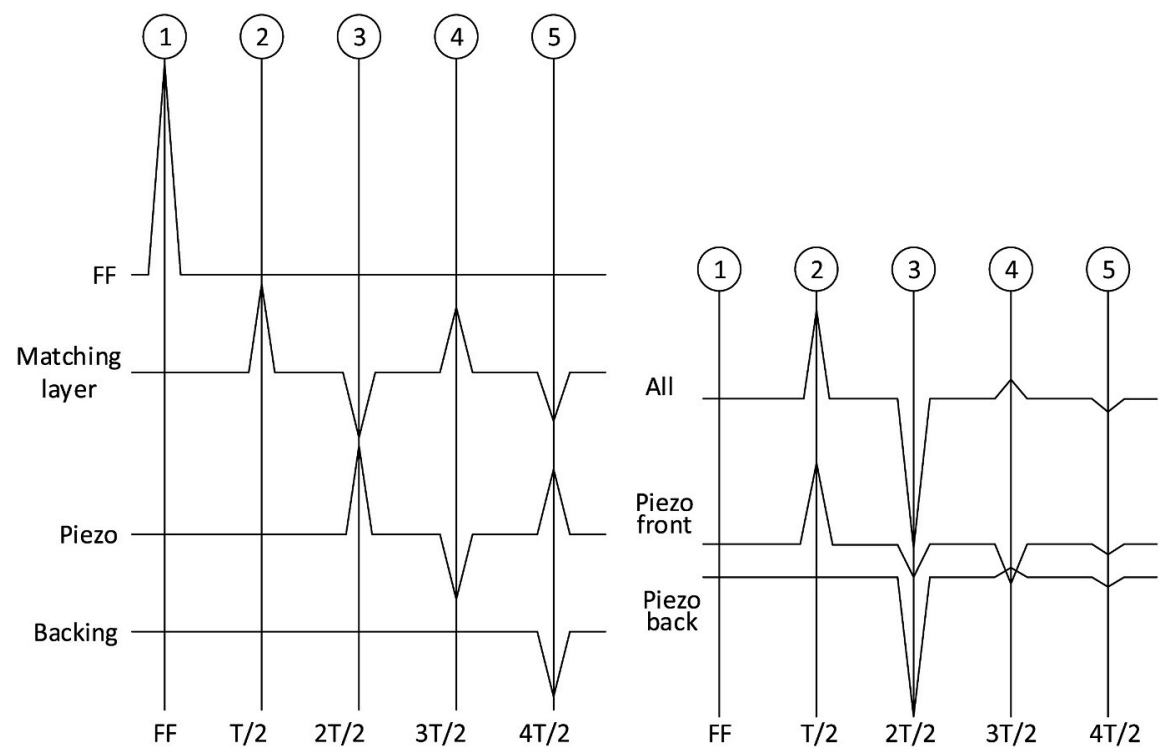

Fig. 37. Signals expected in the reflection (left) and transmission (right) modes for a probe with two matching layers. 

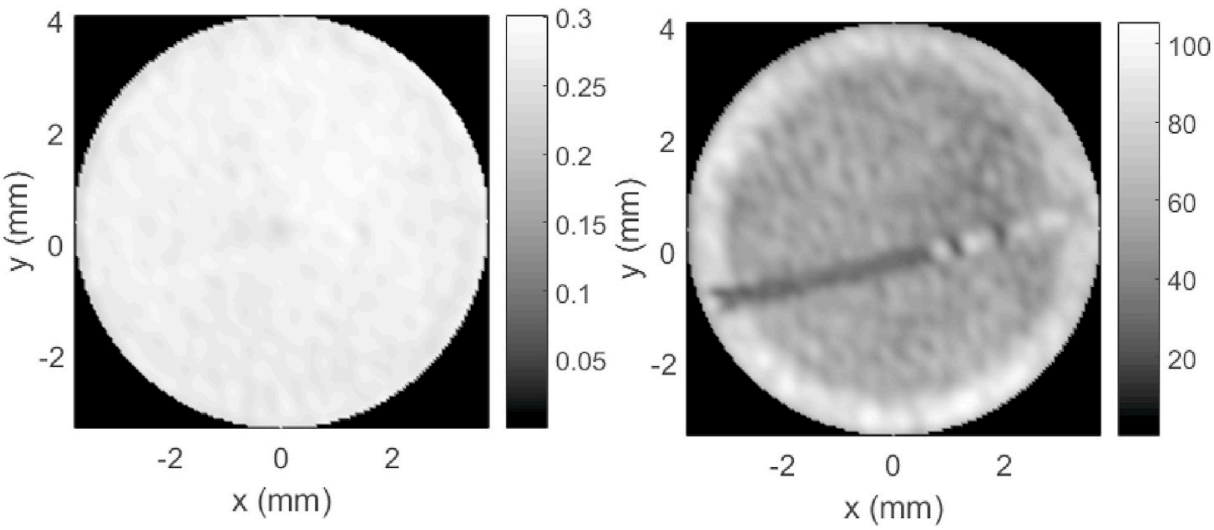

Fig. 38. C-scan of the reflections from the front face (left, grey scale is V) and a second matching layer (right, (grey scale is mV) for the C543-SM probe.
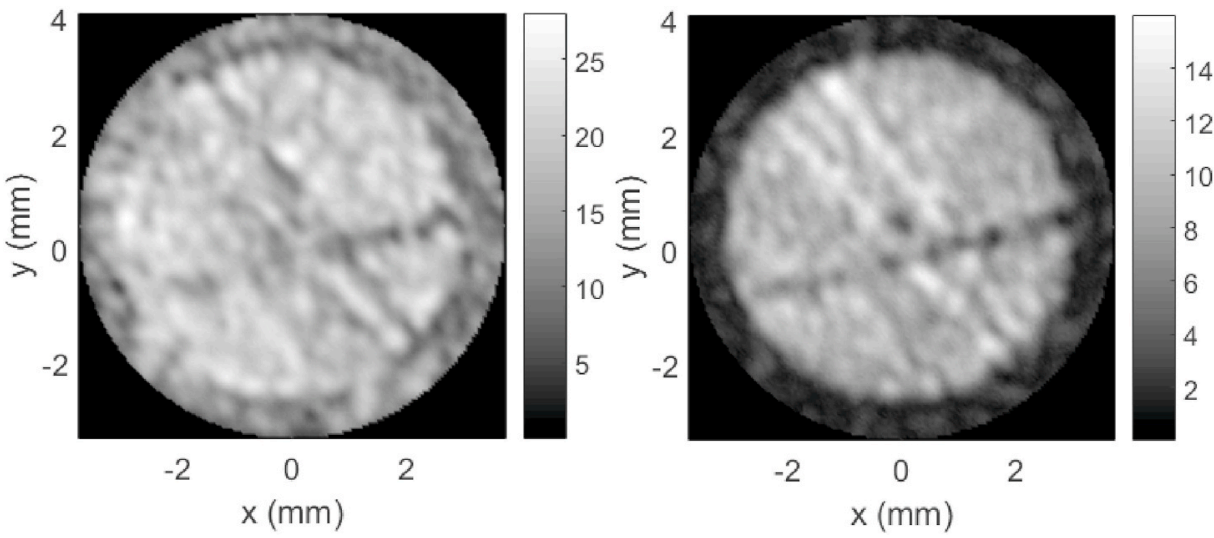

Fig. 39. C-scan of the reflections from the piezoelement (left) and backing (right) for the C543-SM probe (grey scale is mV).
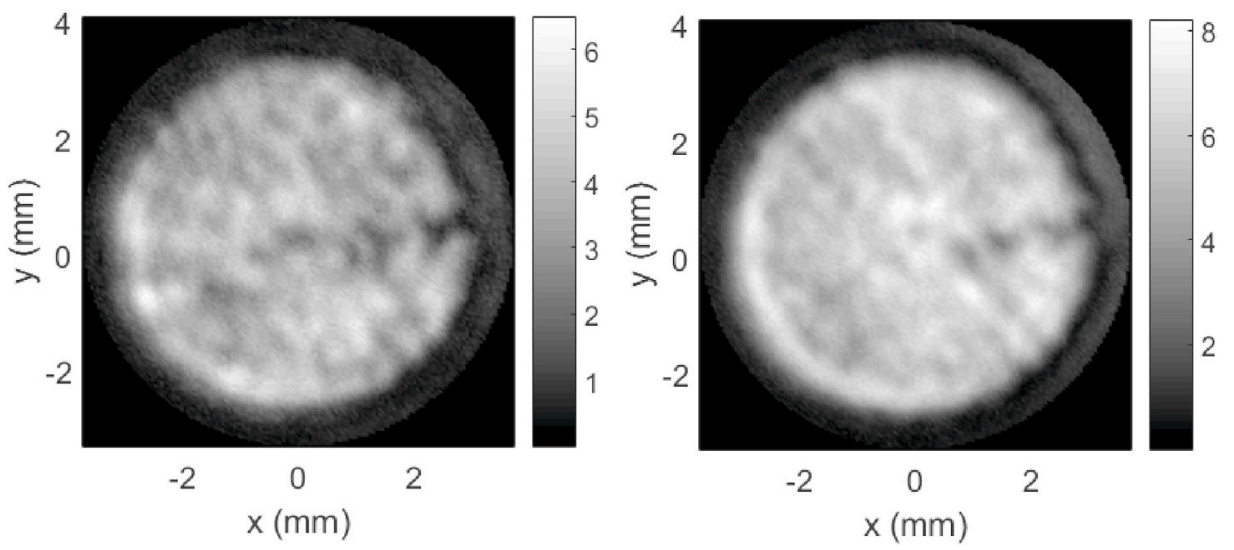

Fig. 40. C-scan in the transmission mode from the piezoelement front (left) and back (right) for a C543-SM probe (grey scale is mV).

Data as Matlab ${ }^{\mathrm{TM}}$ files so the readers can explore the results graphically and see more details.

\section{CRediT authorship contribution statement}

Linas Svilainis: Supervision, Conceptualization, Methodology, Software, Visualization, Writing - original draft. Darius Kybartas: Formal analysis, Validation, Data curation, Writing - review \& editing. Arturas Aleksandrovas: Investigation, Resources. Tomas E.Gomez Alvarez-Arenas: Formal analysis, Writing - review \& editing.

\section{Declaration of competing interest}

The authors declare that they have no known competing financial interests or personal relationships that could have appeared to influence the work reported in this paper.

\section{Acknowledgements}

Funding by grant (DPI2016-78876-R-AEI/FEDER, UE) from the Spanish State Research Agency (AEI) and the European Union, EU is acknowledged. 


\section{Appendix C. Supplementary data}

Supplementary data to this article can be found online at https://doi.org/10.1016/j.ndteint.2020.102360.

\section{Appendix A. Reflection mode signals amplitude analysis}

The analysis of fourth gate signals (section 2, Figs. 6 and 8), obtained in the reflection mode, is presented here. An assumption is checked that in case of impedances assumed in manuscripts, a fourth gate signal (see Figs. 6-8) is prevailed by the reflection at the interface between the piezoelectric element and the backing block. It can be estimated (neglecting losses and diffraction) as a total reflection coefficient:

$R_{\text {Ematch }}=T_{W-M} \cdot R_{M-P Z T}^{3} \cdot R_{M-W}^{2} \cdot T_{M-W}$,

where $T_{W-M}$ is a transmission coefficient for water-matching layer interface, $R_{M-P Z T}$ is the reflection from a matching-piezoelement interface, $R_{P Z T-M}$ is the reflection from the piezoelement-matching interface, $T_{M-W}$ is a transmission coefficient for a matching-water interface. Equation (A.1) can be rewritten using the acoustic impedances:

$R_{\text {Ematch }}=\frac{4 Z_{W}^{2}\left(Z_{P Z T}-Z_{M}\right)^{3} \cdot\left(Z_{W}-Z_{M}\right)^{2}}{\left(Z_{M}+Z_{W}\right)^{4} \cdot\left(Z_{P Z T}+Z_{M}\right)^{3}}$.

An acoustic pulse from the backing layer total reflection coefficient contains the transmission coefficients for water-matching interface $T_{W-M}$, matching-piezoelement interface $T_{M-P Z T}$, piezoelement-matching interface $\left(T_{P Z T-M}\right)$ and reflection from the backing layer $R_{P Z T-B}$ :

$R_{\text {¿back }}=T_{W-M} \cdot T_{M-P Z T} \cdot R_{P Z T-B} \cdot T_{P Z T-M} \cdot T_{M-W}$.

After the application of the acoustic impedances:

$R_{\text {Eback }}=\frac{16 Z_{W}^{2} \cdot Z_{P Z T} \cdot\left(Z_{B}-Z_{P Z T}\right) \cdot Z_{M}}{\left(Z_{M}+Z_{W}\right)^{2} \cdot\left(Z_{P Z T}+Z_{M}\right)^{2} \cdot\left(Z_{B}+Z_{P Z T}\right)}$.

Substituting the above-mentioned acoustic impedances, the reflection from backing is 2.5 times larger than for the multiple refection inside a matching layer $\left(R_{\Sigma \text { back }}=-0.18\right.$ versus $\left.R_{\Sigma \text { match }}=0.07\right)$.

\section{Appendix B. Transmission mode signals amplitude analysis}

The analysis of why the pulse registered by the backwall (section 3, Figs. 11 and 12) is larger than the front face signal is presented here. It is based on a piezoelectric element (with impedance $Z_{2}$ ) loaded by a $Z_{1}$ impedance on the front face and a $Z_{3}$ impedance on the backwall.

The piezoelectric effect in the case of a plane wave propagating along the polarization axis $\mathrm{x}$ denoted as 3 axis is

$\left\{\begin{array}{c}T_{3}=c_{33}^{D} S_{3}-h_{33} D_{3} \\ E_{3}=-h_{33} S_{3}+\beta_{33}^{S} D_{3}\end{array}\right.$

where $T$-stress, $S$ - strain, $E$ - electric field intensity, $D$ - electric displacement, $c^{D}{ }_{33}$ - elastic constant at a constant electric displacement $(D=0), h_{33}-$ piezoelectric constant, $\beta_{33}^{S}$ - component of an inverted dielectric permittivities tensor $\left[\varepsilon^{S}\right]^{-1}$ at a constant $\operatorname{strain}(S=0)$.

In the reception mode the piezoelement vibrates unloaded (no current flows), therefore $\mathrm{D}=0$ and

$\left\{\begin{array}{c}T_{3}=c_{33}^{D} S_{3} \\ E_{3}=-h_{33} S_{3}\end{array}\right.$

The strain along the propagation axis $\mathrm{x}$

$S_{3}=\frac{\partial u}{\partial x}$

where $u$ is a mechanical displacement.

The incident wave propagates from media 1 (acoustic impedance $Z_{1}$, wave number $k_{1}$ ), then it is transmitted to the piezoelectric media $2\left(Z_{2}, k_{2}\right)$, propagates to the backwall and is partially reflected from the interface with backing media $3\left(Z_{3}, k_{3}\right)$. At the interface $1-2$ (front media - piezoelectric) we have a transmitted wave with magnitude $A$. The displacement and strain are

$u_{T(2)}=A \cdot \sin \left(k_{2} x-\omega t\right)$

$S_{3_{-} T(2)}=A k_{2} \cdot \cos \left(k_{2} x-\omega t\right)$

At the interface 2-3 (piezoelectric - backing) if no diffraction and no attenuation exist, the transmitted wave is reflected back

$u_{R(2)}=B \cdot \sin \left(-\left(k_{2} x-\omega t\right)\right)$

$S_{3_{-} R(2)}=-B k_{2} \cdot \cos \left(k_{2} x-\omega t\right)$ 


\section{The reflection coefficient}

$\frac{B}{A}=\frac{Z_{3}-Z_{2}}{Z_{2}+Z_{3}}$

A magnitude of a total strain at the interface 2-3 (back wall) is a sum of strains of the incident $\left(S_{3} T_{(2)}\right)$ and reflected waves

$S_{3 \_\Sigma(2-3)}=A\left(1-\frac{Z_{3}-Z_{2}}{Z_{2}+Z_{3}}\right)$

When a piezoelectric material acoustic impedance is larger than backing $\left(Z_{2}>Z_{3}\right)$, a total strain at the back wall of a piezoelement is larger than the strain on the front wall

$S_{3_{-} T(2)}=A$

Therefore, the electrical pulses from the back wall have a larger amplitude. Substituting the piezoelectric ceramic $\left(Z_{P Z T} \sim 36 \mathrm{MRayl}\right)$ and backing material $\left(Z_{B} \sim 10 \mathrm{MRayl}\right)$ acoustic impedances, a response from the back wall is 1.56 larger than from the front wall.

\section{References}

[1] EN 12668-2. Non-destructive testing - characterization and verification of ultrasonic examination equipment - Part 2: Probes. 2010. 2010 [Bsigroup].

[2] Lopez-Sanchez A, Schmerr L. Determination of an ultrasonic transducer's sensitivity and impedance in a pulse-echo setup. IEEE Trans Ultrason Ferroelectrics Freq Contr 2006;53:2101-12. https://doi.org/10.1109/TUFFC.2006.150.

[3] EN 12668-3. Non-destructive testing - characterization and verification of ultrasonic examination equipment - Part 3: combined equipment. 2013. 2013 [Saiglobal].

[4] Opielinski K, Gudra T. Influence of the thickness of multilayer matching systems on the transfer function of ultrasonic airborne transducer. Ultrasonics 2002;40:465-9. https://doi.org/10.1016/S0041-624X(02)00160-9.

[5] Alvarez-Arenas TEG. Acoustic impedance matching of piezoelectric transducers to the air. IEEE Trans Ultrason Ferroelectrics Freq Contr 2004;51(5):624-33. https:// doi.org/10.1109/TUFFC.2004.1320834.

[6] Engholm M, Stepinski T. Designing and evaluating transducers for narrowband ultrasonic spectroscopy. NDT Int 2007;40:49-56. https://doi.org/10.1016/j. ndteint.2006.07.006.

[7] Ono K. Frequency dependence of receiving sensitivity of ultrasonic transducers and acoustic emission sensors. Sensors 2018;18:3861. https://doi.org/10.3390/ s18113861.

[8] Tomov BG, Diederichsen SE, Thomsen E, Jensen AJ. Characterization of medical ultrasound transducers. IEEE Int Ultrason Symp 2018:1-4. https://doi.org/ 10.1109/ULTSYM.2018.8579798.

[9] Cowell D, Carpenter T, Fisher B, Freear S. In-situ measurement of transducer impedance using AFE active termination through analysis of ultrasound echoes. IEEE Int Ultrason Symp 2017:1-4. https://doi.org/10.1109/ ULTSYM.2017.8092045.

[10] Schmerr LW, Lopez-Sanchez A, Huang R. Complete ultrasonic transducer characterization and its use for models and measurements. Ultrasonics 2006;44: 753-7. https://doi.org/10.1016/j.ultras.2006.05.088.

[11] Silva C, Alvarenga A, Costa-Felix R. Nondestructive testing ultrasonic immersion probe assessment and uncertainty evaluation according to EN 12668-2:2010. IEEE Trans Ultrason Ferroelectrics Freq Contr 2012;59:2338-46. https://doi.org/ 10.1109/TUFFC.2012.2459.

[12] Marhenke T, Sanabria SJ, Chintada BR, Furrer R, Neuenschwander J, Goksel O. Acoustic field characterization of medical array transducers based on unfocused transmits and single-plane hydrophone measurements. Sensors 2019;19:863. https://doi.org/10.3390/s19040863.

[13] Lu J, Greenleaf JF. Evaluation of transducers with near-field scanning of their surfaces. IEEE Int Ultrason Symp 1994:1163-7. https://doi.org/10.1109/ ULTSYM.1994.401793.

[14] Zipser L, Franke H. Laser-scanning vibrometry for ultrasonic transducer development. Sens Actuators, A 2004;110:264-8. https://doi.org/10.1016/j. sna. 2003.10.051.

[15] Lorentsson R, Hosseini N, Johansson JO, Rosenberg W, Stenborg B, Mansson LG, Bath M. Method for automatic detection of defective ultrasound linear array transducers based on uniformity assessment of clinical images - a case study. J Appl Clin Med Phys 2018;19(2):265-74. https://doi.org/10.1002/acm2.12248.

[16] Dudley NJ. Ultrasound probe acceptance testing using the in-air reverberation pattern. Ultrasound Med Biol 2019;45(6):1475-82. https://doi.org/10.1016/j. ultrasmedbio.2019.02.001.
[17] Schindel DW. Air coupled ultrasonic measurements of adhesively bonded multilayer structures. Ultrasonics 1999;37(3):185-200. https://doi.org/10.1016/ S0041-624X(98)00061-4.

[18] Korzeniowski M, Piwowarczyk T, Maev RG. Application of ultrasonic method for quality evaluation of adhesive layers. Arch Civ Mech Eng 2014;14:661-70. https:// doi.org/10.1016/j.acme.2013.10.013.

[19] Thomson WT. Transmission of elastic waves through a stratified solid medium. J Appl Phys 1950;21(89):89-93. https://doi.org/10.1063/1.1699629.

[20] Brekhovskikh LM. Waves in layered media. second ed. New York: Academic Press; 1976 [Elsevier].

[21] Coen S. On the elastic profiles of a layered medium from reflection data. Part 1. Plane-wave sources. J Acoust Soc Am 1981;70:172-5. https://doi.org/10.1121/ 1.386669.

[22] Fantosa F, Symes WW. The determination of a layered acoustic medium via multiple impedance profile inversions from plane wave responses. Geophys J Int 1985;81:175-95. https://doi.org/10.1111/j.1365-246X.1985.tb01357.x.

[23] Lekner J. Matrix methods in reflection and transmission of compressional waves by stratified media. J Acoust Soc Am 1990;87(6):2319. https://doi.org/10.1121/ 1.399077.

[24] Cao W, Qi W. Plane wave propagation in finite 2-2 composites. J Appl Phys 1995; 78(7):4627-32. https://doi.org/10.1063/1.360701.

[25] Svilainis L, Dumbrava V, Chaziachmetovas A, Aleksandrovas A. Pulser for arbitrary width and position square pulse trains generation. IEEE Int Ultrason Symp 2012: 1-4. https://doi.org/10.1109/ULTSYM.2012.0438.

[26] Svilainis L, Dumbrava V, Kybartas D. Evaluation of the ultrasonic preamplifier noise voltage density. J Circ Syst Comput 2014;23(1):1450007. https://doi.org/ 10.1142/S0218126614500078.

[27] Svilainis L, Dumbrava V, Kitov S, Aleksandrovas A, Tervydis P, Liaukonis D. Electronics for ultrasonic imaging system. Elektron Elektrotech 2014;20(7):51-6. https://doi.org/10.5755/j01.eee.20.7.8024.

[28] Brown JL. A Hilbert transform product theorem. Proc IEEE 1986;74:520-1. https://doi.org/10.1109/PROC.1986.13495.

[29] Ulrich TJ. Envelope calculation from the Hilbert transform, technical report. USA: Los Alamos National Laboratory: Los Alamos, NM; 2006 [Google Scholar].

[30] Svilainis L. Review on time delay estimate subsample interpolation in frequency domain. IEEE Trans Ultrason Ferroelectrics Freq Contr 2019;66(11):1691-8. https://doi.org/10.1109/TUFFC.2019.2930661.

[31] Smith SW. Fourier transform properties. In: The scientist and engineer's guide to digital signal processing. second ed. California Technical Pub; 1999 [Analog Devices].

[32] Olofsson T, Stepinski T. Maximum a posteriori deconvolution of sparse ultrasonic signals using genetic optimization. Ultrasonics 1999;37(6):423-32. https://doi. org/10.1016/S0041-624X(99)00019-0.

[33] Svilainis L, Lukoseviciute K, Liaukonis D. Reiterative deconvolution: new technique for time of flight estimation errors reduction in case of close proximity of two reflections. Ultrasonics 2017;76:154-65. https://doi.org/10.1016/j. ultras.2017.01.003.

[34] Ramadas SN, O'Leary RL, Gachagan A. Ultrasonic sensor design for NDE application: design challenges \& considerations. In: Indian national seminar \& exhibition on non-destructive evaluation; 2009. p. 88-91 [Semantic scholar]. 